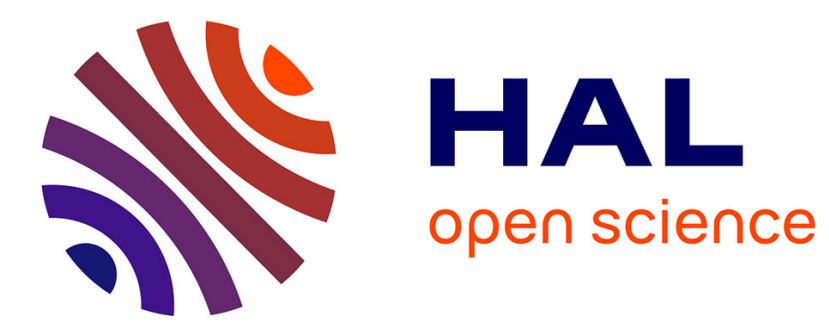

\title{
Theory of temperature-dependent consumer-resource interactions
}

\author{
Alexis Synodinos, B. Haegeman, Arnaud Sentis, José Montoya
}

\section{To cite this version:}

Alexis Synodinos, B. Haegeman, Arnaud Sentis, José Montoya. Theory of temperature-dependent consumer-resource interactions. 2021. hal-03223982

\section{HAL Id: hal-03223982 \\ https://hal.science/hal-03223982}

Preprint submitted on 12 Oct 2021

HAL is a multi-disciplinary open access archive for the deposit and dissemination of scientific research documents, whether they are published or not. The documents may come from teaching and research institutions in France or abroad, or from public or private research centers.
L'archive ouverte pluridisciplinaire HAL, est destinée au dépôt et à la diffusion de documents scientifiques de niveau recherche, publiés ou non, émanant des établissements d'enseignement et de recherche français ou étrangers, des laboratoires publics ou privés. 
1 Title: Theory of temperature-dependent consumer-resource interactions

3 Running head: Temperature-dependence of consumer-resource interactions

5 Keywords: climate change, consumer-resource dynamics, food webs, community stability,

6 biomass distributions, interaction strength, temperature dependence.

$8 \quad$ Article Type: Ideas and Perspectives

9

10 Authors: Alexis D. Synodinos ${ }^{1 *}$, Bart Haegeman ${ }^{1}$, Arnaud Sentis ${ }^{2}$, José M. Montoya ${ }^{1}$

$12{ }^{1}$ Theoretical and Experimental Ecology Station, CNRS, Moulis, 09200, France

13 IINRAE, Aix Marseille Univ., UMR RECOVER, 3275 route Cézanne, 13182 Aix-en-

14 Provence, France.

15

$16 *$ Correspondence to

17 Alexios Synodinos, Theoretical and Experimental Ecology Station, UMR 5321, 2 Route du

18 CNRS, Moulis, 09200, France, Tel : +33 5610405 89, email :

19 alexios.synodinos@sete.cnrs.fr

20

21 Author emails:

22 Bart Haegeman: bart.haegeman@sete.cnrs.fr

$23 \quad$ Arnaud Sentis: arnaud.sentis@inrae.fr

24 José M. Montoya: josemaria.montoyateran@sete.cnrs.fr 
26 Author contributions: ADS, BH, AS and JMM conceived the approach. ADS and BH

27 developed the method, AS and JMM guided its application. ADS led the writing of the 28 manuscript; $\mathrm{BH}$, AS and JMM provided crucial input and guidance throughout the writing 29 process. JMM obtained the funding and managed the project. The authors declare no 30 competing interests.

32 Data accessibility statement: This study produced no new data. Any data used was taken 33 from existing publication and is detailed in the Supplementary Information.

35 Number of words in Abstract: 191

37 Number of words in main text (excluding abstract, figure and table legends, 38 acknowledgments and references): 7,441

39

$40 \quad$ Number of references: 73

41

42 Number of figures: 7

43

44 Number of tables: 1 


\section{Abstract}

48 Changes in temperature affect consumer-resource interactions, which underpin the

49 functioning of ecosystems. However, existing studies report contrasting predictions regarding

50 the impacts of warming on biological rates and community dynamics. To improve prediction 51 accuracy and comparability, we develop an approach that combines sensitivity analysis and

52 aggregate parameters. The former determines which biological parameters impact the

53 community most strongly at a given temperature. The use of aggregate parameters (i.e., 54 maximal energetic efficiency, $\rho$, and interaction strength, $\kappa$ ), that combine multiple biological 55 parameters, increases explanatory power and reduces the complexity of theoretical analyses. 56 We illustrate the approach using empirically-derived thermal dependence curves of biological 57 rates and applying it to consumer-resource biomass ratio and community stability. Based on 58 our analyses, we generate four predictions: 1) resource growth rate regulates biomass 59 distributions at mild temperatures, 2) interaction strength alone determines the thermal 60 boundaries of the community, 3) warming destabilises dynamics at low and mild 61 temperatures only, 4) interactions strength must decrease faster than maximal energetic 62 efficiency for warming to stabilise dynamics. We argue for the potential benefits of directly

63 working with the aggregate parameters to increase the accuracy of predictions on warming 64 impacts on food webs and promote cross-system comparisons.

65 
Introduction

68

69 Temperature strongly regulates consumer-resource interactions that constitute the

70 fundamental blocks of ecosystems (O’Connor et al. 2009; Montoya \& Raffaelli 2010;

71 Petchey et al. 2010; Rall et al. 2012; Amarasekare 2019), and anthropogenic climate change 72 will, in most cases, increase mean temperatures (IPCC 2013). Therefore, understanding and

73 predicting the impacts of warming on consumer-resource interactions has attracted much 74 interest (Vasseur \& McCann 2005; Binzer et al. 2012; Thakur et al. 2017). A breakthrough 75 occurred with the postulation that metabolic rate increases exponentially with temperature, 76 with the slope (often referred to as activation energy) conserved across levels of organisation 77 (Gilooly et al. 2001; Brown et al. 2004). However, activation energies can vary significantly among organisms and biological rates (Dell et al. 2011; Réveillon et al. 2019). In addition, the thermal response curve of biological rates can decrease at high temperatures, producing a unimodal thermal dependence shape (Deutsch et al. 2008; Pörtner \& Farrell 2008; Englund et al. 2011; Uiterwaal \& DeLong 2020). This lack of consensus regarding the exact shape of the temperature-dependence of physiological rates (e.g. ingestion rates), behavioural traits (e.g. consumer search or attack rates) or production (carrying capacity) has contributed to diverging, sometimes contradicting, predictions of how consumer-resource interactions will respond to warming (e.g. Vucic-Pestic et al. 2011; Sentis et al. 2012).

\section{A dual approach to address the divergence in predictions}

89 Two community properties describing important features of the community are consumer-

90 resource biomass ratio and stability, defined with respect to the occurrence of oscillations.

91 Their importance is reflected in their prevalence in the literature on the effects of warming on 
consumer-resource communities and food webs (Rall et al. 2010, 2012; Uszko et al. 2017; Barbier \& Loreau 2019; Bideault et al. 2020). However, predictions vary about the impacts of warming on both these community properties. Biomass ratios have been theorised to increase (Rip \& McCann 2011; Gilbert et al. 2014) or decrease (Vasseur \& McCann 2005) monotonically with warming, though experimentally-derived data have mainly yield unimodal responses (Fussmann et al. 2014; Uszko et al. 2017). Likewise, the effects of warming on stability remain unclear. Using data on specific rates (e.g. consumer ingestion and metabolism), studies have inferred that stability either increases monotonically (Rall et al. 2010, 2012; Vucic-Pestic et al. 2011; Fussmann et al. 2014) or responds unimodally (Sentis et al. 2012; Betini et al. 2019) to warming. Theoretical work on stability, in particular on the mechanisms causing the onset of oscillations, expands decades (Rosenzweig \& MacArthur 1963; May 1972). Vasseur and McCann (2005) showed that warming will destabilise consumer-resource communities when the consumer metabolic rate increases slower than the ingestion rate. Johnson and Amarasekare (2015) demonstrated the pivotal role of the temperature-dependence of carrying capacity - rather than metabolism and ingestion - in determining warming-stability relationships. All these examples demonstrate that the mixed predictions, whether empirically-derived or theoretical, originate from two distinct sources: the different parameters hypothesised to be driving community responses and the thermal dependence shapes of these parameters. To improve the accuracy of predictions regarding the effects of warming on consumer-resource communities, we need to establish which biological parameters drive community properties (biomass distribution, stability) and to acquire a mechanistic understanding of how their thermal dependence shapes affect community properties. 
116 A dual approach utilising sensitivity analysis and the application of aggregate parameters can

117 address both these issues (Fig. 1). On the one hand, sensitivity analysis establishes the

118 parameters that most strongly influence the community property of interest: it quantifies the

119 incremental increase in a response variable with respect to a small incremental increase in a

120 parameter. It has been extensively used in population ecology and demography (Caswell

121 2019), with important applications in applied ecology (Manlik et al. 2018). Since the relative

122 importance of parameters can change along the temperature gradient, a sensitivity analysis

123 allows us to determine the temperatures at which changes in the values of the different

124 parameters have the strongest relative impact (Zhao et al. 2020).

125

126 On the other hand, we can aggregate groups of the primary parameters into fewer,

127 biologically meaningful and empirically measurable quantities. The use of such aggregate

128 parameters reduces the complexity of theoretical analyses, provides a mechanistic

129 interpretation for the difference in predictions and facilitates the comparison among

130 predictions (Barbier \& Loreau 2019; Bideault et al. 2020). Experimentally, replacing multiple

131 measurements of individual parameters with measurements of the aggregates could also

132 restrict the room for divergent findings. The seminal work of Yodzis and Innes (1992)

133 reduced the analysis of consumer-resource interactions to two principal aggregate parameters;

134 consumer maximal energetic efficiency and a measure of resource abundance. A variation of

135 maximal energetic efficiency (termed energetic efficiency) has been widely used by empirical

136 studies. However, rather than being measured directly, it has been derived from

137 measurements of its principle components, i.e., feeding and metabolic rates (Rall et al. 2010;

138 Vucic-Pestic et al. 2011; Sentis et al. 2012). Gilbert et al. (2014) posited that a single

139 aggregate parameter - interaction strength defined as the impact of the consumer on the

140 resource population density - could capture the effects of warming on the stability of 
consumer-resource interactions. However, their approach was based on a type I (nonsaturating) functional response, whereas most consumer-resource species pairs typically produce type II or III (saturating) functional responses (Jeschke et al. 2004). Moreover, the thermal dependence of interaction strength did not match the impact of warming on stability for type II or III functional responses (Uszko et al. 2017), pointing to a more complex relationship between interaction strength, warming and stability. We use two aggregate parameters: the maximal energetic efficiency of the consumer population, defined as the ratio of energetic gains through ingestion with no resource limitation (i.e., maximal energetic gains) over energetic losses associated to metabolic demand (Yodzis \& Innes 1992) and interaction strength, measured as the ratio of resource population density without consumers to resource population density with consumers (Gilbert et al. 2014).

Thus, our dual approach identifies the parameters causing the divergence in predictions through the sensitivity analysis and simplifies complex theoretical explorations and empirical measurements through the two aggregate parameters (Fig. 1). By expressing the sensitivities and the community dynamics in terms of the aggregate parameters, analyses collapse into two dimensions. This creates a simple and mechanistic tool to increase the accuracy and improve comparability of theoretical and empirically-driven predictions on the impact of temperature changes on consumer-resource communities. The dual approach of sensitivity analysis and parameter aggregation is not tailored to a specific model of consumer-resource interactions as both are often used independently for different ecological models (e.g. Barbier et al., add a ref for sensitivity). Here, we apply this general approach to the Rosenzweig-MacArthur model (Rosenzweig \& MacArthur 1963), a model frequently used to study the effects of temperature on consumer-resource interactions (Fussmann et al. 2014; Uszko et al. 2017; Daugaard et al. 2019; Dee et al. 2020). Moreover, our approach can be applied to both static 
166 and dynamic properties of consumer-resource interactions. We illustrate this application

167 using consumer-resource biomass ratio and a stability metric quantifying the proximity to 168 oscillations, respectively; these two variables dominating the literature on the effects of 169 warming on consumer-resource communities (Vasseur \& McCann 2005; Rall et al. 2008; 170 Uszko et al. 2017; Betini et al. 2019). We implement different thermal parameterisations 171 from the literature to elucidate how the relative importance of different parameters and their 172 varying thermal dependence shapes impact predicted effects of temperature on consumer173 resource interactions. Based on our results, we make four predictions that can be theoretically 174 and empirically tested.

175

176 The dual approach

177

178 In this study we illustrate the application of the dual approach (i.e. parameter sensitivity and 179 aggregation) using the Rosenzweig-MacArthur model with a type II functional response 180 (Rosenzweig \& MacArthur 1963). This model describes the rate of change in resource and consumer biomass densities:

182

$183 \quad \frac{d R}{d t}=r\left(1-\frac{R}{K}\right) R-\frac{a R}{1+a h R} C$

184

$185 \frac{d C}{d t}=\left(e \frac{a R}{1+a h R}-m\right) C$

186

$187 R$ and $C$ are the resource and consumer species biomass densities, respectively. Resource 188 growth is logistic, with an intrinsic growth rate, $r$, and carrying capacity, $K$. Resource 189 biomass density is limited by the consumer through a saturating Holling type II functional response with attack rate, $a$, and handling time, $h$. Consumer growth is proportional to the 
191

192

193

194

196

197

198

199

200

201

202

213 For our analyses we assumed dynamics had converged to the stable equilibrium or the limit

214 cycle and determined the coexistence equilibria and the biomass ratio analytically (SI 1).

215 Equilibrium means zero rate of change for both consumer and resource population biomasses.

assimilated consumed biomass, with $e$ the dimensionless assimilation efficiency; losses occur due to metabolic costs, $m$. Below we present the formulas most relevant to our study; an extensive analysis of the model is available in the supplementary information (SI 1). We chose a type II response due to its prevalence in many natural consumer-resource interactions (Jeschke et al. 2004), though our approach works for the general form of the functional response (SI 2). Additionally, the functional response can be defined with respect to attack rate and handing time, $f(R)=\frac{a R}{1+a h R}$, or maximum consumption rate, $J$, and half-saturation density, $R_{0}, f(R)=\frac{J R}{R_{0}+R}(\mathrm{SI} 3)$.

\section{Stability and aggregate parameters}

As demonstrated through the 'paradox of enrichment' (Rosenzweig 1971), the RosenzweigMacArthur model produces population cycles (oscillations) with increasing energy fluxes (Rip \& McCann 2011). Therefore, the coexistence equilibrium can be stable or unstable, where dynamics oscillate around the unstable equilibrium (i.e., a limit cycle). The switch from stable to unstable dynamics occurs at a Hopf bifurcation. Theoretical studies have analysed this qualitative change (Yodzis \& Innes 1992; Vasseur \& McCann 2005; Amarasekare 2015) because these distinct stability regimes translate to different temporal dynamics, with oscillations leading to greater variability over time. We applied a stability metric that quantifies the tendency of the dynamics to oscillate (Johnson \& Amarasekare 2015). 
216 For the limit cycle, this yields the unstable equilibrium which is approximately equal to the

217 time-averaged biomass values along the limit cycle. Thus, we set equations (1) and (2) to

218 zero, solved to yield the coexistence equilibrium and retrieved the biomass ratio:

219

$220 \mathcal{B}=\frac{C_{S}}{R_{S}}=\operatorname{er} \frac{a K(e-m h)-m}{a m K(e-m h)}$

222 In the model analysis we observed certain repeated parameter groupings (i.e., aggregate 223 parameters) governed the dynamics (SI 1). Such aggregates have been previously used for the analysis of the Rosenzweig-MacArthur model (Yodzis \& Innes 1992; Vasseur \& McCann 2005). The aggregates we selected represent ecological mechanisms which can be empirically measured. These are maximal energetic efficiency, $\rho=\frac{e}{m h}$, and interaction strength,

$\kappa=\operatorname{ahK}\left(\frac{e}{m h}-1\right)$. A closer look at $\rho$ and $\kappa$ elucidates their biological meaning. $\frac{1}{h}$ is the saturation value of the functional response and thus represents the maximum consumption rate, $J$. Hence, $\rho$ can be written as:

230

$$
\rho=\underbrace{e}_{\begin{array}{c}
\text { consumer } \\
\text { assimilation } \\
\text { efficiecny }
\end{array}} * \underbrace{J}_{\begin{array}{c}
\text { maximum } \\
\text { consumption rate }
\end{array}} * \begin{array}{c}
\text { consumer } \\
\text { metabolic } \\
\text { loss }
\end{array}_{\frac{1}{m}}^{\text {energetic loss }}=\frac{\text { maximal energetic gain }}{\text { merimes }}
$$

$\rho$ quantifies the energetic gain-to-loss ratio of the consumer population biomass assuming its maximum feeding rate is realised (i.e. unlimited resources). $\rho$ was introduced by Yodzis and

234 Innes (1992) as a key aggregate parameter to understand food web dynamics. In empirical 235 studies, a variant of $\rho$ termed energetic efficiency, $y$, has been often applied (Rall et al. 2010; 236 Sentis et al. 2012, 2017). Unlike $\rho, y$ is a function of the full functional response term and 
237 hence also depends on resource density, $y=\frac{e * f(R)}{m}$, where $f(R)=\frac{a R}{1+a h R}$ at a specified 238 resource density, $R$.

239

240 The second aggregate parameter, $\kappa$, can be rewritten in terms of the resource population 241 density:

242

$$
\kappa=\operatorname{ahK}\left(\frac{e}{m h}-1\right)=\frac{K}{R_{S}}
$$

243

$244 \kappa$ is the ratio of the resource equilibrium density without consumers (carrying capacity) to the 245 resource equilibrium density with consumers. $\kappa$ quantifies the effect of the consumer 246 population on the resource population and measures interaction strength (Berlow et al. 1999;

247 Gilbert et al. 2014).

248

249 Using $\rho$ and $\kappa$ we determine the conditions for positive resource (eq. 4) and consumer (eq. 5)

250 densities, as well as the Hopf bifurcation (eq. 6) (SI 1):

251

$252 \rho>1$

253

$254 \kappa>1$

255

$256 \kappa-\rho-1=0$ 
$258 \kappa>1$ requires that $\rho>1$ (SI 1 ). Hence, $\kappa>1$ defines the consumer feasibility boundary. We do

259 not consider stochastic extinctions which may occur due to large-amplitude oscillations when

260 population biomass reaches very low values.

261

262 To determine stability, we adjusted the metric of Johnson and Amarasekare (2015) so that it 263 vanished at the Hopf bifurcation (SI 4). This metric, $\mathcal{S}$, defines stability solely in relation to 264 the Hopf bifurcation.

$$
\mathcal{S}=-\frac{(\kappa-\rho-1)}{\rho-1}
$$

$267 \mathcal{S}>0$ corresponds to a stable equilibrium and $\mathcal{S}<0$ to oscillations.

Sensitivity analysis

271 We performed a sensitivity analysis of the biomass ratio $(B)$ and the stability metric $(\mathcal{S})$ with 272 respect to the original model parameters (i.e., $r, a, h, e$ and $m$ ). A sensitivity analysis 273 quantifies the effect of an infinitesimal change in a parameter on the response variable.

274 Typically, while one parameter is being perturbed, all others are assumed to remain constant 275 and correlations between the parameters are not explicitly considered. However, this 276 approach can be applied in cases of correlated parameter change without a loss of accuracy. 277 As many environmental conditions (e.g. temperature) induce correlated changes in the 278 parameters, then the sensitivity of the response variable (e.g. $B$ or $\mathcal{S}$ ) with respect to the 279 environmental conditions can be reconstructed by combining the sensitivities of the 280 individual parameters (SI 5.1). Different types of sensitivity indices exist such as simple 281 sensitivity and elasticity (Manlik et al. 2018; Caswell 2019). Here we used elasticity for 
282 biomass ratio $(B)$ and an adjusted elasticity for stability $(\mathcal{S})$ (SI 5.1), both dimensionless to

283 facilitate direct comparisons between parameter sensitivities.

284

285 Elasticity is a proportional sensitivity, quantifying how a relative change in a parameter translates into a relative change in the variable; otherwise known as the log-scaled sensitivity

287 (Manlik et al. 2018). Thus, the elasticity of $B$ with respect to parameter $x$ is given by:

288

$$
\partial_{x} \mathcal{B}=\frac{\frac{\partial \mathcal{B}}{\mathcal{B}}}{\frac{\partial x}{x}}=\frac{\partial \ln (\mathcal{B})}{\partial \ln (x)}
$$

289

290 If $\partial_{x} B=1$, a relative increase of $10 \%$ in parameter $x$ causes a relative increase of $10 \%$ in

291 variable $B$. Conversely, $\partial_{x} B=-1$ implies that a relative increase of $10 \%$ in parameter $x$ results

292 in relative decrease of $10 \%$ in $B$.

293

294 For the sensitivity of the stability metric, $\mathcal{S}$, we used a variation of the elasticity. We defined

295 the sensitivity of $\mathcal{S}$ as the incremental change in $\mathcal{S}$ induced by a relative change in parameter

$296 x$. Our adjustment was possible due to $\mathcal{S}$ being dimensionless and it prevents sensitivities 297 from diverging to infinity close to the Hopf bifurcation without altering the outcome of our 298 analysis (SI 5.1).

299

$$
\partial_{x} \mathcal{S}=\frac{\partial \mathcal{S}}{\frac{\partial x}{x}}=\frac{\partial \mathcal{S}}{\partial \ln (x)}
$$


$\partial_{x} \mathcal{S}=1$ implies that a relative increase in parameter $x$ of $10 \%$ translates into an absolute

302 increase of 0.1 in $\mathcal{S}$ and has a stabilising effect. If, conversely, $\partial_{x} \mathcal{S}=-1$, the same relative

303 increase in $x$ would lead to decrease of 0.1 in $\mathcal{S}$ with a destabilising effect.

305 The magnitude and sign of each sensitivity determine how strongly and in what direction the

306 parameter perturbation impacts the variable, respectively. We used the magnitudes to rank the

307 relative importance of all parameters. The sign provided qualitative information regarding the

308 direction of change (increasing or decreasing the response variable).

310 All sensitivities could be expressed in terms of $\rho$ and $\kappa$. Hence, sensitivities are fully

311 determined in a plane with of $\rho$ and $\kappa$ as axes (Fig. SI 5.1, 5.2). In this $\rho$ - $\kappa$ plane the

312 sensitivities of all parameters can be ranked by magnitude, splitting the parameter space into

313 regions where different parameters have the highest, second highest, etc. sensitivity

314 magnitude. Here, we present figures where the regions are determined by the top-two ranked

315 sensitivities; we do not portray changes in the rankings of the lowest sensitivities (see Fig. SI

3165.4 and SI 5.5 for the complete biomass ratio and stability metric regions, respectively). The

317 biomass ratio and stability metric have different sensitivity expressions and, therefore,

318 produced different regions. For each variable, the regions remain fixed irrespective of the

319 parameterisation used, because the sensitivity expressions stem from the model equations.

320 The $\rho$ - $\kappa$ plane provides additional information, such as the feasibility boundary (eq. 5) and

321 the position of the Hopf bifurcation (eq. 6). 
325 To demonstrate the impacts of different parameter thermal dependencies, we implemented

326 temperature parameterisations from the literature. Maintenance respiration rates, $m$, have

327 been shown to increase exponentially with temperature (Brown et al. 2004). The Arrhenius

328 equation is most often used to describe this thermal dependence (Vasseur \& McCann 2005;

329 Sentis et al. 2017; Uszko et al. 2017). However, the thermal response curves of resource

330 growth rate, $r$, attack rate, $a$, and handling time, $h$, have been represented either through the

331 Arrhenius equation (Vasseur \& McCann 2005; Binzer et al. 2016) or as unimodal functions

332 (Amarasekare 2015; Uszko et al. 2017; Zhang et al. 2017; Uiterwaal \& DeLong 2020; Zhao

333 et al. 2020). Carrying capacity, $K$, and consumer assimilation efficiency, $e$, have a less clear

334 connection to temperature (Uszko et al. 2017; Dee et al. 2020). We selected two

335 parametrisations related to the ongoing debate surrounding the importance of including the

336 decreasing part of the biological rates beyond the optimal temperature (Pawar et al. 2016)

337 and used these as an illustrative comparison. The 'unimodal' model had a unimodal

338 parameterisation for $r, a$ (both hump-shaped) and $h$ (U-shaped), the Arrhenius equation for $m$

339 (increasing), and constant $K$ and $e$ (Uszko et al. 2017). We compared this to a 'monotonic'

340 parameterisation where all thermal dependencies ( $r, a, m$ increasing; $h, K$ decreasing) follow

341 the Arrhenius equation and $e$ is constant (Fussmann et al. 2014). Following this comparison,

342 we plotted four additional parameterisations from the literature onto the $\rho$ - $\kappa$ plane to broaden

343 the comparison and demonstrate the simplicity of applying the approach to empirically-

344 derived measurements. These consisted of two similar monotonic parameterisations (Vucic-

345 Pestic et al. 2011; Binzer et al. 2016), one where only a was hump-shaped (Sentis et al.

346 2012) and one which though monotonic, included some distinctive thermal dependencies -

347 exponentially increasing $K(T)$ and $e(T)$ and constant $h$ (Archer et al. 2019). We provide a

348 description of the studies and details of their parameterisations in the supplementary material 349 (SI 6). 
351 We should note that not all parameterisations included the resource growth rate, $r$, so the

352 biomass ratio could not be calculated in these cases. However, we could calculate $\rho$ and $\kappa$

353 and, hence, the biomass ratio elasticities for all parameterisations. Thus, we could determine

354 how biomass ratio sensitivities to individual parameters changed with warming regardless of

355 the actual biomass ratio values. By including studies which had not measured resource

356 growth or estimated the biomass ratio we broadened the scope of the comparison of the

357 biomass ratio sensitivities. Though this does not represent an exhaustive list of

358 parameterisations, we were restricted to parameterisations which could be used to parametrise

359 the Rosenzweig-MacArthur model with a type II response and whose available parameters

360 could yield $\rho$ and $\kappa$. 'Mild' and 'extreme' temperatures, as well as 'close' or 'far' from

361 consumer extinction, are defined relative to each parameterisation's temperature range and

362 feasibility boundaries, respectively. The feasible temperature range was determined by

363 interaction strength $(\kappa>1)$ with the temperature extremes corresponding to the point of

364 consumer extinction. This condition assumes that resources have a broader thermal range

365 than consumers (e.g., Rose \& Caron 2007; West \& Post 2016). If resources go extinct at

366 temperatures consumers could withstand, then the feasibility boundary becomes dependent

367 on resource growth rate, $r$ (Amarasekare 2015). The parameterisations we present come from

368 ectotherms, where environmental temperatures correspond to the organisms' temperatures.

369 However, our approach can be transferred to endotherms as it does not depend on a specific

370 thermal parametrisation.

372 Sensitivities depend on proximity to thermal boundaries 
376 We analytically obtained four groups of biomass ratio elasticity magnitudes, $\partial_{e} B=\left|\partial_{m} B\right|$, $377 \partial_{K} B=\partial_{a} B, \partial_{r} B$ and $\partial_{h} B . e$ and $m$ always have the largest elasticity and hence the strongest 378 relative impact on the biomass ratio $\left(\partial_{e} B=\left|\partial_{m} B\right|>\partial_{K} B, \partial_{a} B, \partial_{r} B,\left|\partial_{h} B\right|\right.$, Table 1$)$. Increasing $e$ 379 increases the biomass ratio $\left(\partial_{e} B>0\right)$, increasing $m$ reduces it $\left(\partial_{m} B<0\right) . K$ and $a$ have equal 380 and positive elasticities $\left(\partial_{K} B=\partial_{a} B>0\right)$, both increasing the biomass ratio. The elasticity of $r$ 381 is constant, $\partial_{r} B=1$; a directly proportional positive effect on $B$. Increasing $h$ reduces the 382 biomass ratio, $\partial_{h} B<0$. These are general results, independent of any model parameterisation 383 (with temperature or otherwise) following directly from the Rosenzweig-MacArthur model's 384 equations.

Biomass ratio: high r elasticity far from thermal boundaries

Both the 'unimodal' and 'monotonic' temperature parameterisations produced a unimodal biomass ratio thermal dependence (Fig. 2). The unimodal parameterisation induced thermal boundaries to the community at both low $\left(1{ }^{\circ} \mathrm{C}\right)$ and high $\left(33^{\circ} \mathrm{C}\right)$ temperatures (Fig. 2a). The biomass ratio exceeded 1 for most temperatures (higher consumer than resource biomass),

392 peaked at $14^{\circ} \mathrm{C}$ around $B \approx 5.5$ and decreased rapidly to 0 as it approached both thermal boundaries (low and high temperature extremes). The biomass ratio of the monotonic parameterisation (Fig. $2 b$ ) increased with warming from low temperatures, peaked at $B \approx 0.19$, before decreasing to 0 at high temperatures $\left(27.5^{\circ} \mathrm{C}\right)$. The two parameterisations are derived from different systems, hence the different temperature ranges.

398 In both parameterisations, sensitivity to $e$ and $m$ was highest throughout (Fig. 2c, d) - as expected from the analytical findings. Elasticities were split into two groups at mild 
temperatures: $e, m$ and $r$ had the highest elasticity with $\partial_{e} B=\left|\partial_{m} B\right| \approx \partial_{r} B=1$, while $a, h$ and $K$

401 elasticities were very low. Approaching the temperature extremes all elasticities besides $\partial_{r} B$

402 diverged; $\partial_{h} B$ diverged faster than $\partial_{K} B=\partial_{a} B$ in the unimodal parameterisation (Fig. 2c),

403 while the opposite occurred in the monotonic one (Fig. 2d).

405 Expressing the elasticities in terms of $\rho$ and $\kappa$ (Table 1) reduces the sensitivity analysis to two 406 dimensions. Ranking the elasticity magnitudes creates distinct regions in the $\rho$ - $\kappa$ plane which 407 correspond to different elasticity ranking orderings and provide a mechanistic overview of 408 which elasticities dominate where (Fig. 3a). $e$ and $m$ always have the highest elasticity, so the three regions reflect changes in the second highest-ranked elasticity. Regions adjacent to consumer extinction ( $\kappa=1$ ) have high sensitivity to either $h$ (red region) or to $a$ and $K$ (yellow region). $r$ elasticity is highest in the region farthest from consumer extinction (orange region).

412 The two temperature parameterisations were mapped onto this plane by calculating their $\rho$ 413 and $\kappa$ values (Fig $3 \mathrm{~b}$ and $\mathrm{c}$ ). Despite the two trajectories being markedly different, both 414 occupied the region where $r$ ranked second highest for most temperatures. The unimodal 415 parameterisation produced a unimodal trajectory, crossing the consumer extinction threshold 416 at low and high temperatures (Fig. 3b). The monotonic parameterisation's trajectory 417 converged monotonically towards consumer extinction with increasing temperature (Fig. 3c).

419 All other parameterisations from the literature also occupied the region of high $r$ elasticity for 420 most temperatures, far from their thermal boundaries (Fig. 4). Three monotonic 421 parameterisations produced monotonic trajectories (Fig. 4a, b, c) which started in the region 422 of high $r$ elasticity and converged monotonically towards consumer extinction $(\kappa=1)$. With a 423 hump-shaped thermal dependence of attack rate, a unimodal trajectory emerged (Fig. 4d). At 424 low temperatures it occupied the region of high $r$ elasticity but moved away from consumer 
425

426

427

428

429

430

431

432

433

434

436

437

438

439

440

441

442

443

444

445

446 The unimodal temperature parameterisation exhibited oscillations $(\mathcal{S}<0)$ over most

extinction. With further warming, the trajectory switched direction and followed the same path as the monotonic parameterisations, crossing the consumer extinction boundary. A unimodal thermal dependence for attack rate (hump-shaped) and handling time (U-shaped) (Fig. 4e), induced extinctions at low and high temperatures imposing a unimodal trajectory. Unlike all previous parameterisations, the trajectory crossed the extinction threshold in the region of high $h$ elasticity. The final parameterisation, though monotonic, yielded unimodal trajectories (Fig. 4f). A monotonically increasing $K(T)$ (as opposed to decreasing in the other monotonic parameterisations and constant in the unimodal ones) initially forced the trajectory away from consumer extinction, albeit within the region of high $r$ elasticity. Consumer energetic efficiency $\rho$ decreased, pushing consumers towards extinction, thus forcing an abrupt decline towards the consumer boundary.

\section{Stability most sensitive either to $e$ and $m$ or to $a$ and $K$}

Similarly to the biomass ratio, the analytical approach for the stability sensitivities yielded results conserved independently of the temperature parameterisations (Table 1): equal sensitivity magnitudes pairwise for $e$ and $m$ and for $a$ and $K$ (i.e., $\left|\partial_{e} \mathcal{S}\right|=\partial_{m} \mathcal{S}$ and $\left|\partial_{K} \mathcal{S}\right|=\left|\partial_{a} \mathcal{S}\right|$ ), negative stability sensitivities of $e, a$ and $K\left(\partial_{e} \mathcal{S}, \partial_{a} \mathcal{S}, \partial_{K} \mathcal{S}<0\right)$ implying they destabilise dynamics, a positive sensitivity of $m\left(\partial_{m} \mathcal{S}>0\right)$ indicating a stabilising effect. $h$ can be either stabilising or destabilising and $r$ does not affect the stability regime $\left(\partial_{r} \mathcal{S}=0\right)$. temperatures (Fig. 5a). Only at low and high thermal extremes did dynamics briefly stabilise prior to consumer extinction. The monotonic temperature parametrisation produced oscillations at low temperatures $(\mathcal{S}<0)$, crossed a Hopf bifurcation at $17^{\circ} \mathrm{C}$ and dynamics 
were stable $(\mathcal{S}>0)$ thereafter (Fig. 5 b). In both cases, stability close to consumer extinction was most sensitive to consumer assimilation efficiency, $e$, and metabolism, $m,\left(\left|\partial_{e} \mathcal{S}\right|=\partial_{m} \mathcal{\delta}\right)$

452 followed by handling time, $h$ (Fig. 5c, d). Moving away from the thermal boundaries, attack 453 rate, $a$, and carrying capacity, $K$, increased in relative importance. Furthest away from the 454 thermal boundaries, stability was most sensitive to changes $a$ and $K$, followed by $h$. Even though $\partial_{h} \mathcal{S}$ did not rank highest in any temperature range, it was a close second both at the temperature extremes (second to $\left|\partial_{e} \mathcal{S}\right|=\partial_{m} \mathcal{S}$ ) or furthest away from them (second to $\left.\left|\partial_{K} \mathcal{S}\right|=\left|\partial_{a} \mathcal{S}\right|\right)$. Additionally, $h$ switched from destabilising at mild temperatures $\left(\partial_{h} \mathcal{S}<0\right)$ to stabilising $\left(\partial_{h} \mathcal{\delta}>0\right)$ close to the temperature extremes (Fig. 5c, d, Fig. S5.2).

The $\rho$ - $\kappa$ plane for the stability metric was split into four regions; in two regions closest to consumer extinction, $\left|\partial_{e} \mathcal{S}\right|=\partial_{m} \mathcal{S}$ were the largest sensitivities (Fig. 6a, red and yellow regions) and in the two regions furthest from consumer extinction, $\left|\partial_{K} \mathcal{S}\right|=\left|\partial_{a} \mathcal{S}\right|$ ranked highest (Fig. 6a, green and blue regions). Additionally, the Hopf bifurcation (Fig. 6a, dashed curve) split the plane into stable equilibrium and oscillation regions. Corresponding to the general findings, stability in the two reference ('unimodal' and 'monotonic') parameterisations was most sensitive to changes in $e$ and $m$ at the thermal extremes - close to consumer extinction and to $a$ and $K$ at milder temperatures - far from consumer extinction (Fig. 6b, c). The unimodal trajectory occupied the region of oscillations for most temperatures, crossing the Hopf bifurcation twice close to consumer extinction, once at low and once at high

470 temperatures (Fig. 6b, blue region). The monotonic trajectory started in the region of oscillations and moved into the stable region with warming, crossing the Hopf bifurcation far 472 from the thermal extreme (Fig. 6c, yellow region). 
476 Plotting the other temperature parameterisations' trajectories onto the $\rho-\kappa$ plane reproduced

477 the same patterns with respect to the stability metric's sensitivity (Fig. 7): stability was most

478 sensitive to $e$ and $m$ at the thermal extremes and to $a$ and $K$ far from the extremes.

479 Significantly, the trajectories revealed the impact of the thermal dependence shape of 480 individual parameters on the warming-stability relationship. In three monotonic 481 parameterisations, warming stabilised the dynamics (Fig. 7 a, b, c). In the cases, when 482 oscillations did take place, these occurred at low temperatures (Fig 7a resident prey, b, c) and 483 dynamics crossed the Hopf bifurcation far from the thermal boundary. In the case with two 484 enrichment levels (Fig. 7c), the high enrichment scenario required higher temperatures to 485 stabilise the dynamics. For the unimodal trajectory with hump-shaped attack rate (Fig. 7d), warming at low temperatures pushed the dynamics towards (low enrichment) or deeper into (high enrichment) the region with oscillations (i.e., destabilised dynamics). Here too, the destabilising impact of enrichment was evident. However, further warming switched the 489 direction of the trajectory. Subsequently, both $\rho$ and $\kappa$ decreased. $\kappa$ declined much faster, 490 forcing the dynamics towards the stable region and eventually consumer extinction. Both the 491 switch in the trajectory direction and the Hopf bifurcation (high enrichment scenario) 492 occurred at mild temperatures, in the region of high $a$ and $K$ sensitivity. In the 493 parameterisation with both $a$ (hump-shaped) and $h$ (U-shaped) unimodal (Fig. 7e), the Hopf 494 bifurcation occurred close to the thermal boundaries, where $\kappa$ increased (low temperatures) or 495 decreased (high temperatures) much faster than $\rho$. The dynamics were oscillatory for most 496 temperatures, with the switch in the trajectory's direction occurring in the region of highest 497 sensitivity to $a$ and $K$. The final parameterisation's trajectories were characterised by a 498 negative relationship between $\rho$ and $\kappa$ (Fig. 7f). Driven by the positive thermal dependence of 
499

500

501

502

503

504

505

506

507

508

509

510

511

512

513

carrying capacity, warming increased $\kappa$ and destabilised dynamics which oscillated for most temperatures.

\section{Discussion}

Research on the impacts of warming on consumer-resource interactions has yielded mixed results (Vasseur \& McCann 2005; Englund et al. 2011; Rall et al. 2012; Gilbert et al. 2014; Uszko et al. 2017). Resolving this debate and improving predictions has become even more pressing as most ecosystems face increased temperatures (Easterling et al. 2000; Walther et al. 2002; Root et al. 2003; Parmesan 2006). Here, we developed an approach to improve and simplify predictions on the impacts of warming on consumer-resource interactions. This approach integrates two pathways: (1) a sensitivity analysis to identify the key biological parameters whose variations have the largest relative impact on community properties at a given temperature, and (2) aggregate parameters (maximal energetic efficiency, $\rho$, and interaction strength, $\kappa$ ) to increase explanatory power. We used the Rosenzweig-MacArthur model with a type II functional response, and applied the approach to consumer-resource biomass ratio and a stability metric quantifying the propensity for oscillations (Johnson \& Amarasekare 2015). Therefore, our insights apply to study systems well-described by the Rosenzweig-MacArthur model. Our analyses revealed that the relative significance of different parameter groupings is determined by the proximity of the consumer to its thermal boundaries. We, further, elucidated how differences in the shape of the thermal dependence curves of individual parameters qualitatively impact predictions. We used empiricallyderived thermal dependence curves of biological parameters from the literature to illustrate this. 
524 We focus our discussion on the formulation of four testable predictions arising from our 525 results. For each prediction, we present its implications and rationale. Then, we discuss the 526 empirical measurement of the aggregate parameters and present important subtleties and 527 potential extensions of our approach.

Prediction 1: Resource growth rate regulates biomass distribution at mild temperatures

531 Implications: We showed that the relative dominance of consumer assimilation efficiency, 532 metabolism and resource growth rate in driving changes in biomass distributions should 533 manifest itself in any consumer-resource community far from its feasibility boundaries, 534 assuming these communities are well-described by the Rosenzweig-MacArthur model (Fig. 535 3a). Due to the agreement about the thermal dependence of metabolism (Rall et al. 2010; 536 Fussmann et al. 2014; Uszko et al. 2017) and the negligible -if any- change of assimilation 537 efficiency with warming (Dell et al. 2011), differences in the thermal performance curve of 538 resource growth rate will strongly impact biomass ratio predictions. Therefore, improved 539 predictions about the impacts of warming on biomass distributions at mild temperatures necessitate the accurate description of the thermal dependence of resource growth rate.

542 Reasoning: Far from the community thermal boundaries, consumer assimilation efficiency, 543 metabolism and resource growth rate always had the greatest elasticity with an almost equal 544 relative impact on biomass ratio $\left(\partial_{e} B=\left|\partial_{m} B\right| \approx \partial_{r} B=1\right.$, Fig. $2 \mathrm{c}, \mathrm{d}$ and Fig. S5.1). Increasing 545 metabolism reduced biomass ratios (Table 1), which is likely to be a universal response 546 across ecosystems, given the positive exponential dependence of metabolism on temperature 547 across organisms (Gilooly et al. 2001; Brown et al. 2004; Rall et al. 2012 but see Ehnes et al. 548 2011). Conversely, assimilation efficiency increased biomass ratios but has either been 
assumed to be unaffected by temperature changes (Vasseur \& McCann 2005; Sentis et al. 2017; Uszko et al. 2017) or has yielded a weak temperature-dependence (Wurtsbaugh \&

551 Davis 1977; Handeland et al. 2008; Lang et al. 2017; Daugaard et al. 2019) with negligible 552 change compared to other parameters. Increasing resource growth rate also increased the 553 biomass ratio. However, evidence on the shape of resource growth's thermal response 554 remains inconclusive: it can either increase exponentially with temperature (Savage et al. 555 2004) or decrease abruptly beyond the thermal optimum (Dannon et al. 2010; Thomas et al. 556 2012). Since the biomass ratio is directly proportional to the resource growth rate $\left(\partial_{r} B=1\right.$, 557 Table 1), it will be strongly affected by the values and shape of the resource growth rate 558 thermal performance curve. Given the consensus surrounding the temperature-dependence of metabolism and the minor scale of potential change in assimilation efficiency with temperature, our findings emphasise the significance of correctly parameterising the resource growth rate when aiming to predict biomass distribution changes due to warming at mild 562 temperatures.

Prediction 2: Interaction strength determines consumer survival with increasing temperatures.

567 Implications: If resources have a broader thermal range compared to consumers (Rose \& 568 Caron 2007; West \& Post 2016), the thermal boundaries of the community can be determined 569 by measuring solely the thermal dependence of interaction strength, $\kappa$. This quantity - the 570 ratio of the resource equilibrium density without consumers (carrying capacity) to the 571 resource equilibrium density with consumers - can be determined experimentally (Berlow et 572 al. 2004) or through observations, facilitating predictions and cross-system comparisons 573 thereof. 
575 Reasoning: Close to the consumer extinction boundary, consumer survival becomes 576 extremely sensitive to all parameters apart from resource growth (Fig. 2, S5.1), making 577 accurate predictions challenging. Currently, consumer survival has been inferred through 578 energetic efficiency - the effective energetic gain of consumers at a certain resource density 579 - which requires determining the thermal dependence of the functional response (Vucic580 Pestic et al. 2011; Archer et al. 2019). Not only is the functional response's thermal 581 dependence hotly contested (Uszko et al. 2017; Uiterwaal \& DeLong 2020), but this 582 uncertainty will be exacerbated by its extremely high sensitivity at the community's thermal 583 boundaries. We showed there exists an alternative, empirically more direct and theoretically 584 more robust metric to determine consumer survival, and hence community feasibility. 585 Interaction strength — the relative values of resource equilibrium without and with consumers (Berlow et al. 1999, 2004; Gilbert et al. 2014) — provides the necessary condition

587 for consumer survival $(\kappa>1)$, when resources are thermal generalists compared to consumers.

588 This provides an accurate threshold and represents a measurable quantity that can be standardised across experimental designs and study systems (Berlow et al. 2004).

\section{Prediction 3: Warming reduces community stability at low and mild temperatures}

593 Implications: This prediction rests on important assumptions: that resources have a broader 594 thermal range, that organisms currently experience temperatures below their optima (Pawar 595 et al. 2016) and that the functional response is of type II with a unimodal thermal dependence 596 (Rall et al. 2012; Sentis et al. 2012; Kuiters 2013; West \& Post 2016; Uszko et al. 2017;

597 Uiterwaal \& DeLong 2020). We deem these assumptions realistic based on the literature; 
598

599

600

601 Reasoning: Stability in the context of consumer-resource interactions has predominantly

602 referred to a qualitative distinction between stable and oscillating dynamics (Rosenzweig \&

603 MacArthur 1963; Yodzis \& Innes 1992; Vasseur \& McCann 2005). We based our analysis on

604

605

606

607

608

609

610

611

612

613

614

615

616

617

therefore, we argue that consumer-resource interactions at low and mild temperatures will be destabilised by warming. At higher temperatures, warming should always enhance stability.

\section{MacArthur 1963; Yodzis \& Innes 1992; Vasseur \& McCann 2005). We based our analysis on} an adjusted stability metric which quantifies the tendency of dynamics to oscillate (Johnson \& Amarasekare 2015, SI 4). When comparing existing temperature parameterisations, we found that in most monotonic parameterisations (increasing metabolism and attack rate, decreasing handling time and carrying capacity, assimilation efficiency constant), warming always (i.e., monotonically) stabilised dynamics (Fig. 7a, b, c). The single exception arose when warming and carrying capacity increased simultaneously, which destabilised dynamics (Fig. 7f). Carrying capacity has been described as a proxy for enrichment and its destabilising effect has been established whether independently of temperature (Rosenzweig 1971) or as antagonistic to warming (Binzer et al. 2016). When at least one parameter in the functional response had a unimodal thermal dependence (i.e., hump-shaped attack rate or U-shaped handling time), this yielded a unimodal warming-stability relationship (Fig. 7d, e). Significantly, the divergence between the unimodal and (most) monotonic parameterisations in the predicted effect of warming on stability manifested itself at low or mild, rather than high temperatures (Fig. 6,7). This pattern originates in the impact of the parameters with unimodal thermal dependencies on stability. Attack rate is destabilising (Table 1, McCann 2011). Thus, a hump-shaped thermal dependence of attack rate destabilises dynamics with warming below the thermal optimum and stabilises dynamics beyond it. Handling time is stabilising close to the thermal extremes (Fig. 6c, S5.2). A U-shaped handling time will rapidly decrease with warming from low temperatures, which is strongly destabilising; a 
623

624

625

626

627

628

629

630

631

632

633

634

635

636

638

639

640

641

642

643

644

645 Reasoning: Decreasing energetic efficiency or interaction strength have been considered 646 647

corresponding steep increase at high temperatures produces a strong stabilising effect. Thus, warming at high temperatures will always be stabilising. However, at lower temperatures, unimodal and monotonic thermal dependencies produce contrasting warming-stability relationships. Therefore, the thermal dependence shape of the functional response combined with the temperatures currently experienced by communities relative to their optimal temperature will determine the impact of warming on stability (Betini et al. 2019).

Prediction 4: Warming stabilises dynamics only when interaction strength decreases faster than maximal energetic efficiency

Implications: The combination of $\rho$ - the energetic gain-to-loss ratio of consumers given unlimited resources — and $\kappa$ - interaction strength — accurately describes the warmingstability relationship with no recourse to the thermal dependence shapes of individual parameters, the current temperatures relative to the thermal optima, or the proximity to the thermal boundaries of the community. Therefore, differential responses of resources and consumers to warming (Dell et al. 2014) will be encompassed by the thermal dependence of the aggregates - assuming the consumer-resource system is well-described by the Rosenzweig-MacArthur model. The Hopf bifurcation condition (eq. 6) dictates that $\kappa$ should decrease faster than $\rho$ for warming to stabilise consumer-resource interactions. Thus, measuring $\rho$ and $\kappa$ directly can increase the accuracy of warming-stability predictions and simplify cross-system comparisons.

equivalent to increasing stability (Rall et al. 2008, 2010; Sentis et al. 2012). Thus, estimates of consumer energetic efficiency or interaction strength based on empirically-derived thermal 
648 dependence curves of individual rates (e.g. ingestion rate, attack rate, metabolic rate) have

649 been used to infer the impacts of warming on stability (Rall et al. 2010, 2012; Vucic-Pestic et 650 al. 2011; Fussmann et al. 2014). However, this raises two significant issues. On the one hand, 651 even subtle changes in the thermal dependence shapes of individual parameters can yield all 652 possible outcomes (Amarasekare 2015). On the other hand, reducing the analysis of stability 653 to a single aggregate parameter has limitations. Gilbert et al. (2014) described the warming654 stability relationship with a single aggregate, interaction strength, but their approach was 655 based on a type I functional response and its predictions do not work well in type II or III 656 scenarios (Uszko et al. 2017). Johnson and Amarasekare (2015) and Amarasekare (2015) 657 attained a single aggregate parameter to reduce the complexity of their explorations; 658 however, this lacks descriptive power of the dynamics close to the community's thermal 659 boundaries (SI 4). Our analysis in the $\rho-\kappa$ plane suggests that stability cannot be reduced to a 660 single aggregate parameter nor does a decrease in either one or both of $\rho$ and $\kappa$ suffice to 661 stabilise dynamics. In fact, both $\rho$ and $\kappa$ can decrease with warming while dynamics become 662 destabilised. A stabilising effect of warming requires not only a concurrent reduction in $\rho$ and $663 \kappa$, but also the latter to decrease faster. Critically, both $\rho$ and $\kappa$ represent biological quantities 664 which can be consistently measured across study systems.

Working with the aggregate parameters

668 Working directly with the two aggregate parameters, maximal consumer energetic efficiency, $669 \rho$, and interaction strength, $\kappa$, can simplify empirical measurements and improve the accuracy 670 of theoretical predictions, particularly for field data and experiments, as we argue below. To determine the thermal dependence of maximal consumer energetic efficiency and interaction

672 strength, one can measure consumer population growth given unlimited resources and 
673

674

675

676

677

678

679

680

681

682

683

684

685

resource population density in presence and absence of consumers at different temperatures, respectively. These measurements can be performed in the lab and the field. Interaction strength is commonly determined in field experiments where consumers are excluded (Berlow et al. 2004; Wootton \& Emmerson 2005; Novak 2010; Estes et al. 2011). Consumer energetic gain-to-loss ratio under effectively unlimited resources is more rarely estimated. However, it can be derived from consumer population net growth and metabolism and mortality, quantities measured commonly in the field and in the lab (Hanson \& Peters 1984; Stemberger \& Gilbert 1985; Lampert et al. 1986). Moreover, confounding factors in field measurements of the population-level aggregates should generate less uncertainty compared to that of measuring multiple individual parameters, where uncertainty propagates and often generates large uncertainty in model predictions (e.g. Sentis et al. 2015). Therefore, working directly with the aggregate parameters can be both simpler and lead to more accurate predictions in the field. On the other hand, measuring the individual parameters in the lab has well-established protocols and a history of reliable outputs, with measurements requiring only short-term experiments as opposed to the aggregates.

The choice between measuring the aggregate or the individual parameters will be informed by the questions and objectives of each study. The aggregates describe population-level mechanisms of consumer-resource interactions, while the individual parameters correspond to physiological or behavioural processes of individual organisms scaled up to the population level. As we argued, the aggregates can provide more accurate predictions for field measurements whereas individual parameters can be accurately measured in the lab. This does raise the question whether measurements in a controlled laboratory environment can represent noisier conditions in the field. It would be useful to compare directly measured 
697

698

699

700

701

702

703

704

705

706

707

708

709

710

711

712

713 Regarding the stability of consumer-resource interactions, the $\rho$ - $\kappa$ plane helped visualise the 714

715

716

717

718

719

720

aggregate parameters to aggregate parameter values derived from the individual parameters to determine how well predictions based on individual rates capture the dynamics of the system. Regardless of the choice, our approach provides the tools for both pathways: studies working with individual parameters will benefit from identifying the most important parameters to measure, while aggregate parameter datapoints can be directly mapped onto the $\rho-\kappa$ landscape.

\section{Subtleties and extensions}

The sensitivity analysis quantified the sensitivity of the model variables to infinitesimal parameter changes. Therefore, applying its insights to data should take into consideration the scales of parameters in the temperature range of interest and potential uncertainties in the parameter estimates (Manlik et al. 2018, Fig. Sx). Hence, our argument for the reduced significance of the thermal dependence of assimilation efficiency in driving changes in biomass distributions, despite its high sensitivity. stabilising effect of a type III functional response (Fig. S2.1), which has both theoretical and empirical support (Sarnelle \& Wilson 2008; Kalinkat et al. 2013; Uszko et al. 2017; Daugaard et al. 2019). For the type II response, the defining role of the functional response (attack rate) and the carrying capacity has been widely documented (Rosenzweig 1971; Amarasekare 2015; Johnson \& Amarasekare 2015; Binzer et al. 2016); we add the important caveat that this is the case only far from consumer extinction (Fig. 6a). 
721 Finally, relaxing certain assumptions can extend our approach. Considering the scenario

722 where the consumer has a broader thermal niche relative to that of the resource will make the

723 thermal limits of coexistence dependent on resource growth (Amarasekare 2015).

724 Considering the extinction of populations with very low abundances to account for stochastic

725 extinctions can define a realisable coexistence range within the feasible parameter space.

726 Breaking down the original model parameters (e.g. handling time includes the handling and

727 ingestion of prey) could facilitate our understanding of the role of more fundamental

728 physiological processes in the dynamics. Finally, climate change will lead to stronger

729 fluctuations in temperatures (IPCC 2013), which have been shown to alter predictions in

730 consumer-resource dynamics (Vasseur et al. 2014; Dee et al. 2020). This makes the inclusion

731 of temperature variability an important next step.

732

\section{Conclusions}

735 Warming will have significant, but as yet uncertain impacts on consumer-resource 736 interactions which underpin the structure and functioning of ecosystems. We presented an 737 approach that will help to improve the accuracy of predictions and reconcile divergent results

738 by facilitating cross-system comparisons. This approach first determines the parameters 739 whose variations have the largest effect on community properties. Second, it simplifies 740 analyses to a two-dimensional plane of mechanistically tractable aggregate parameters; 741 maximal consumer energetic efficiency and interaction strength. Applying it to consumer742 resource biomass ratio and stability, we showed that close to the consumer extinction 743 boundary (i.e., at temperature extremes) both variables are most sensitive to changes in 744 consumer assimilation efficiency and metabolism. Far from the boundary (i.e., mild 745 temperatures), biomass ratio is most sensitive to resource growth rate, consumer assimilation 
746 efficiency and metabolism. This yielded our first prediction, that resource growth rate

747 regulates biomass distributions at mild temperatures. The consensus around the thermal 748 dependence of metabolism and the limited potential impact of warming on assimilation

749 efficiency, underscore the importance of correctly measuring the thermal dependence of

750 resource growth rate. Using the two aggregate parameters also simplified the study of 751 important properties of consumer-resource interactions. From this followed our second 752 prediction, that the thermal boundaries of the community are defined by interaction strength 753 alone. In terms of stability, we demonstrated that a unimodal thermal dependence of attack 754 rate or handling time alters predictions of warming-stability relationships below the thermal 755 optimum, where many organisms may be currently living. Hence our third prediction, that 756 initial increases in mean temperatures will destabilise consumer-resource interactions. 757 Significantly, our approach elucidates how the thermal dependence of stability can be 758 comprehensively characterised by maximal energetic efficiency and interaction strength 759 values. This produced our fourth prediction; a faster reduction of interaction strength than of 760 maximal energetic efficiency with warming is necessary for dynamics to stabilise. Finally, we 761 demonstrated the potential for targeted experiments to measure the thermal dependencies of 762 maximal energetic efficiency and interaction strength to improve predictions. Ultimately, we

763 show that any temperature parameterisation fitted to the Rosenzweig-MacArthur model can

764 be mapped onto the aggregate parameter plane, revealing its stability landscape, providing a 765 mechanistic interpretation for its predictions and allowing for the cross-system comparison of 766 these predictions.

\section{Acknowledgments}


770

771

772

773

774

775

776

777

778

779

780

781

782

783

784

785

786

787

788

789

790

791

792

793

We thank three anonymous reviewers and the editor for their constructive feedback. This research is supported by the FRAGCLIM Consolidator Grant, funded by the European Research Council under the European Union's Horizon 2020 research and innovation programme (Grant Agreement Number 726176), and by the "Laboratoires d'Excellences (LABEX)" TULIP (ANR-10-LABX-41).

\section{References}

Amarasekare, P. (2015). Effects of temperature on consumer-resource interactions. J. Anim. Ecol., 84, 665-679.

Amarasekare, P. (2019). Effects of Climate Warming on Consumer-Resource Interactions: A Latitudinal Perspective. Front. Ecol. Evol., 7.

Archer, L.C., Sohlström, E.H., Gallo, B., Jochum, M., Woodward, G., Kordas, R.L., et al. (2019). Consistent temperature dependence of functional response parameters and their use in predicting population abundance. J. Anim. Ecol., 88, 1670-1683.

Barbier, M. \& Loreau, M. (2019). Pyramids and cascades: a synthesis of food chain functioning and stability. Ecol. Lett., 22, 405-419.

Berlow, E.L., Navarrete, S.A., Briggs, C.J., Power, M.E. \& Menge, B.A. (1999). Quantifying Variation in the Strengths of Species Interactions. Ecology, 80, 2206.

Berlow, E.L., Neutel, A.M., Cohen, J.E., De Ruiter, P.C., Ebenman, B., Emmerson, M., et al. (2004). Interaction strengths in food webs: Issues and opportunities. J. Anim. Ecol.

Betini, G.S., Avgar, T., McCann, K.S. \& Fryxell, J.M. (2019). Temperature triggers a non linear response in resource-consumer interaction strength. Ecosphere, 10.

Bideault, A., Galiana, N., Zelnik, Y.R., Gravel, D., Loreau, M., Barbier, M., et al. (2020). Thermal mismatches in biological rates determine trophic control and biomass 
distribution under warming. Glob. Chang. Biol., gcb.15395.

796 Binzer, A., Guill, C., Brose, U. \& Rall, B.C. (2012). The dynamics of food chains under climate change and nutrient enrichment. Philos. Trans. R. Soc. B Biol. Sci., 367, 29352944.

Binzer, A., Guill, C., Rall, B.C. \& Brose, U. (2016). Interactive effects of warming, 800 eutrophication and size structure: impacts on biodiversity and food-web structure. Glob. Chang. Biol., 22, 220-227.

802

Brown, J.H., Gillooly, J.F., Allen, A.P., Savage, V.M. \& West, G.B. (2004). TOWARD A METABOLIC THEORY OF ECOLOGY. Ecology, 85, 1771-1789.

Caswell, H. (2019). Sensitivity Analysis: Matrix Methods in Demography and Ecology. Demographic Research Monographs. Springer International Publishing, Cham.

Dannon, E.A., Tamò, M., van Huis, A. \& Dicke, M. (2010). Functional response and life history parameters of Apanteles taragamae, a larval parasitoid of Maruca vitrata. BioControl, 55, 363-378.

Daugaard, U., Petchey, O.L. \& Pennekamp, F. (2019). Warming can destabilize predatorprey interactions by shifting the functional response from Type III to Type II. J. Anim. Ecol., 88, 1575-1586.

Dee, L.E., Okamtoto, D., Gårdmark, A., Montoya, J.M. \& Miller, S.J. (2020). Temperature

Dell, A.I., Pawar, S. \& Savage, V.M. (2011). Systematic variation in the temperature dependence of physiological and ecological traits. Proc. Natl. Acad. Sci. U. S. A., 108, $10591-10596$.

Dell, A.I., Pawar, S. \& Savage, V.M. (2014). Temperature dependence of trophic interactions are driven by asymmetry of species responses and foraging strategy. J. Anim. Ecol., 83, 

$70-84$.

821

Deutsch, C.A., Tewksbury, J.J., Huey, R.B., Sheldon, K.S., Ghalambor, C.K., Haak, D.C., et al. (2008). Impacts of climate warming on terrestrial ectotherms across latitude. Proc. Natl. Acad. Sci., 105, 6668-6672.

824 Easterling, D.R., Meehl, G.A., Parmesan, C., Changnon, S.A., Karl, T.R. \& Mearns, L.O. (2000). Climate extremes: observations, modeling, and impacts. Science, 289, 20682074.

Ehnes, R.B., Rall, B.C. \& Brose, U. (2011). Phylogenetic grouping, curvature and metabolic scaling in terrestrial invertebrates. Ecol. Lett., 14, 993-1000.

829 Englund, G., Öhlund, G., Hein, C.L. \& Diehl, S. (2011). Temperature dependence of the 830 functional response. Ecol. Lett., 14, 914-921.

831 Estes, J.A., Terborgh, J., Brashares, J.S., Power, M.E., Berger, J., Bond, W.J., et al. (2011). Trophic downgrading of planet earth. Science (80-. ).

833 Fussmann, K.E., Schwarzmüller, F., Brose, U., Jousset, A. \& Rall, B.C. (2014). Ecological stability in response to warming. Nat. Clim. Chang., 4, 206-210.

835 Gilbert, B., Tunney, T.D., McCann, K.S., DeLong, J.P., Vasseur, D.A., Savage, V., et al. (2014). A bioenergetic framework for the temperature dependence of trophic interactions. Ecol. Lett., 17, 902-914.

838 Gilooly, J.F., Brown, J.H., Geoffrey, B.W., Savage, V.M. \& Charnov, E.L. (2001). Effects of 839 Size and Temperature on Metabolic Rate. Science (80-. )., 293, 2248-2251.

840 Handeland, S.O., Imsland, A.K. \& Stefansson, S.O. (2008). The effect of temperature and fish size on growth, feed intake, food conversion efficiency and stomach evacuation rate of Atlantic salmon post-smolts. Aquaculture, 283, 36-42. and profundal macrobenthos biomass in lakes. Can. J. Fish. Aquat. Sci., 41, 439-445. 
845

846

847

848

849

850

851

852

853

854

855

856

857

858

859

860

861

862

863

864

865

866

867

868

869

IPCC, 2013: Climate Change 2013: The Physical Science Basis. Contribution of Working Group I to the Fifth Assessment Report of the Intergovernmental Panel on Climate Change [Stocker, T.F., D. Qin, G.-K. Plattner, M. Tignor, S.K. Allen, J. Boschung, A. Nauels, Y. Xia, V. Bex and P.M. Midgley (eds.)]. Cambridge University Press, Cambridge, United Kingdom and New York, NY, USA, 1535 pp.Jeschke, J.M., Kopp, M. \& Tollrian, R. (2004). Consumer-food systems: Why type I functional responses are exclusive to filter feeders. Biol. Rev. Camb. Philos. Soc.

Jeschke, J.M., Kopp, M. \& Tollrian, R. (2004). Consumer-food systems: Why type I functional responses are exclusive to filter feeders. Biol. Rev. Camb. Philos. Soc.

Johnson, C.A. \& Amarasekare, P. (2015). A metric for quantifying the oscillatory tendency of consumer-resource interactions. Am. Nat., 185, 87-99.

Kalinkat, G., Schneider, F.D., Digel, C., Guill, C., Rall, B.C. \& Brose, U. (2013). Body masses, functional responses and predator-prey stability. Ecol. Lett., 16, 1126-1134.

Kuiters, A. t. (2013). Diversity-stability relationships in plant communities of contrasting habitats. J. Veg. Sci., 24, 453-462.

Lampert, W., Fleckner, W., Rai, H. \& Taylor, B.E. (1986). Phytoplankton control by grazing zooplankton: A study on the spring clear water phase. Limnol. Oceanogr., 31, 478490.

Lang, B., Ehnes, R.B., Brose, U. \& Rall, B.C. (2017). Temperature and consumer type dependencies of energy flows in natural communities. Oikos, 126, 1717-1725.

Manlik, O., Lacy, R.C. \& Sherwin, W.B. (2018). Applicability and limitations of sensitivity analyses for wildlife management. J. Appl. Ecol., 55, 1430-1440.

May, R.M. (1972). Limit cycles in predator-prey communities. Science (80-. )., 177, 900902.

McCann, K.S. (2011). Food Webs (MPB-50)| Princeton University Press. Monograpsh Pop 
870

871

872

873

874

875

876

877

878

879

880

881

882

883

884

885

886

887

888

889

890

891

892 893

894

Bio. Available at: https://press.princeton.edu/books/paperback/9780691134185/foodwebs-mpb-50. Last accessed 10 September 2020.

Montoya, J.M. \& Raffaelli, D. (2010). Climate change, biotic interactions and ecosystem services. Philos. Trans. R. Soc. B Biol. Sci., 365, 2013-2018.

Novak, M. (2010). Estimating interaction strengths in nature: experimental support for an observational approach. Ecology, 91, 2394-2405.

O’Connor, M.I., Piehler, M.F., Leech, D.M., Anton, A. \& Bruno, J.F. (2009). Warming and Resource Availability Shift Food Web Structure and Metabolism. PLoS Biol., 7, e1000178.

Parmesan, C. (2006). Ecological and Evolutionary Responses to Recent Climate Change. Annu. Rev. Ecol. Evol. Syst., 37, 637-669.

Pawar, S., Dell, A.I., Savage, V.M. \& Knies, J.L. (2016). Real versus artificial variation in the thermal sensitivity of biological traits. Am. Nat., 187, E41-E52.

Petchey, O.L., Brose, U. \& Rall, B.C. (2010). Predicting the effects of temperature on food web connectance. Philos. Trans. R. Soc. B Biol. Sci., 365, 2081-2091.

Pörtner, H.O. \& Farrell, A.P. (2008). Ecology: Physiology and climate change. Science (80-. ).

Rall, B., Guill, C. \& Brose, U. (2008). Food-web connectance and predator interference dampen the paradox of enrichment. Oikos, 117, 202-213.

Rall, B.C., Brose, U., Hartvig, M., Kalinkat, G., Schwarzmüller, F., Vucic-Pestic, O., et al. (2012). Universal temperature and body-mass scaling of feeding rates. Philos. Trans. $R$. Soc. B Biol. Sci., 367, 2923-2934.

Rall, B.Ö.C., Vucic-Pestic, O., Ehnes, R.B., EmmersoN, M. \& Brose, U. (2010). Temperature, predator-prey interaction strength and population stability. Glob. Chang. Biol., 16, 2145-2157. 
895 Réveillon, T., Rota, T., Chauvet, É., Lecerf, A. \& Sentis, A. (2019). Repeatable inter 896 individual variation in the thermal sensitivity of metabolic rate. Oikos, 128, 1633-1640. 897 Rip, J.M.K. \& McCann, K.S. (2011). Cross-ecosystem differences in stability and the 898 principle of energy flux. Ecol. Lett., 14, 733-740.

899 Root, T.L., Price, J.T., Hall, K.R., Schneider, S.H., Rosenzweig, C. \& Pounds, J.A. (2003). $900 \quad$ Fingerprints of global warming on wild animals and plants. Nature, 421, 57-60.

901 Rose, J.M. \& Caron, D.A. (2007). Does low temperature constrain the growth rates of 902 heterotrophic protists? Evidence and implications for algal blooms in cold waters. 903 Limnol. Oceanogr., 52, 886-895.

904 Rosenzweig, M.L. (1971). Paradox of Enrichment: Destabilization of Exploitation $905 \quad$ Ecosystems in Ecological Time. Science (80-. )., 171, 385-387.

906 Rosenzweig, M.L. \& MacArthur, R.H. (1963). Graphical Representation and Stability 907 Conditions of Predator-Prey Interactions. Am. Nat., 97, 209-223.

908 Sarnelle, O. \& Wilson, A.E. (2008). Type III Functional Response in Daphnia. Ecology, 89, $909 \quad 1723-1732$.

910 Savage, V.M., Gilloly, J.F., Brown, J.H., Charnov, E.L. \& Charnov, E.L. (2004). Effects of 911 body size and temperature on population growth. Am. Nat., 163, 429-41.

912 Sentis, A., Binzer, A. \& Boukal, D.S. (2017). Temperature-size responses alter food chain 913 persistence across environmental gradients. Ecol. Lett., 20, 852-862.

914 Sentis, A., Hemptinne, J.L. \& Brodeur, J. (2012). Using functional response modeling to 915 investigate the effect of temperature on predator feeding rate and energetic efficiency. $916 \quad$ Oecologia, 169, 1117-1125.

917 Sentis, A., Morisson, J. \& Boukal, D.S. (2015). Thermal acclimation modulates the impacts 918 of temperature and enrichment on trophic interaction strengths and population dynamics. 919 Glob. Chang. Biol., 21, 3290-3298. 
920 Stemberger, R.S. \& Gilbert, J.J. (1985). Body Size, Food Concentration, and Population

921 Growth in Planktonic Rotifers. Ecology, 66, 1151-1159.

922 Thakur, M.P., Künne, T., Griffin, J.N. \& Eisenhauer, N. (2017). Warming magnifies 923 predation and reduces prey coexistence in a model litter arthropod system. Proc. R. Soc. $924 \quad$ B Biol. Sci., 284, 20162570.

925 Thomas, M.K., Kremer, C.T., Klausmeier, C.A. \& Litchman, E. (2012). A global pattern of 926 thermal adaptation in marine phytoplankton. Science (80-. )., 338, 1085-1088.

927 Uiterwaal, S.F. \& DeLong, J.P. (2020). Functional responses are maximized at intermediate 928 temperatures. Ecology.

929 Uszko, W., Diehl, S., Englund, G. \& Amarasekare, P. (2017). Effects of warming on 930 predator-prey interactions - a resource-based approach and a theoretical synthesis. Ecol. $931 \quad$ Lett., 20, 513-523.

932 Vasseur, D.A., DeLong, J.P., Gilbert, B., Greig, H.S., Harley, C.D.G., McCann, K.S., et al. 933 (2014). Increased temperature variation poses a greater risk to species than climate $934 \quad$ warming. Proc. R. Soc. B Biol. Sci., 281.

935 Vasseur, D.A. \& McCann, K.S. (2005). A mechanistic approach for modeling temperature936 dependent consumer-resource dynamics. Am. Nat., 166, 184-98.

937 Vucic-Pestic, O., Ehnes, R.B., Rall, B.C. \& Brose, U. (2011). Warming up the system: higher 938 predator feeding rates but lower energetic efficiencies. Glob. Chang. Biol., 17, 1301$939 \quad 1310$.

940 Walther, G.-R., Post, E., Convey, P., Menzel, A., Parmesan, C., Beebee, T.J.C., et al. (2002). 941 Ecological responses to recent climate change. Nature, 416, 389-395.

942 West, D.C. \& Post, D.M. (2016). Impacts of warming revealed by linking resource growth 943 rates with consumer functional responses. J. Anim. Ecol., 85, 671-680.

944 Wootton, J.T. \& Emmerson, M. (2005). Measurement of Interaction Strength in Nature. 
946 Wurtsbaugh, W.A. \& Davis, G.E. (1977). Effects of temperature and ration level on the 947 growth and food conversion efficiency of Salmo gairdneri, Richardson. J. Fish Biol., 11, $948 \quad 87-98$.

949 Yodzis, P. \& Innes, S. (1992). Body size and consumer-resource dynamics. Am. Nat., 139, $950 \quad 1151-1175$.

951 Zhang, L., Takahashi, D., Hartvig, M. \& Andersen, K.H. (2017). Food-web dynamics under 952 climate change. Proc. R. Soc. B Biol. Sci., 284, 20171772.

953 Zhao, Q., Liu, S. \& Niu, X. (2020). Effect of water temperature on the dynamic behavior of 954 phytoplankton-zooplankton model. Appl. Math. Comput., 378, 125211. 
Tables and Figures

956

957 Table 1 . Sensitivities of the biomass ratio $\left(\partial_{x} B\right)$ and of the stability metric $\left(\partial_{x} \mathcal{S}\right)$ with respect

958 to the six original model parameters. All sensitivities are expressed in terms of $\rho$ and $\kappa$.

959

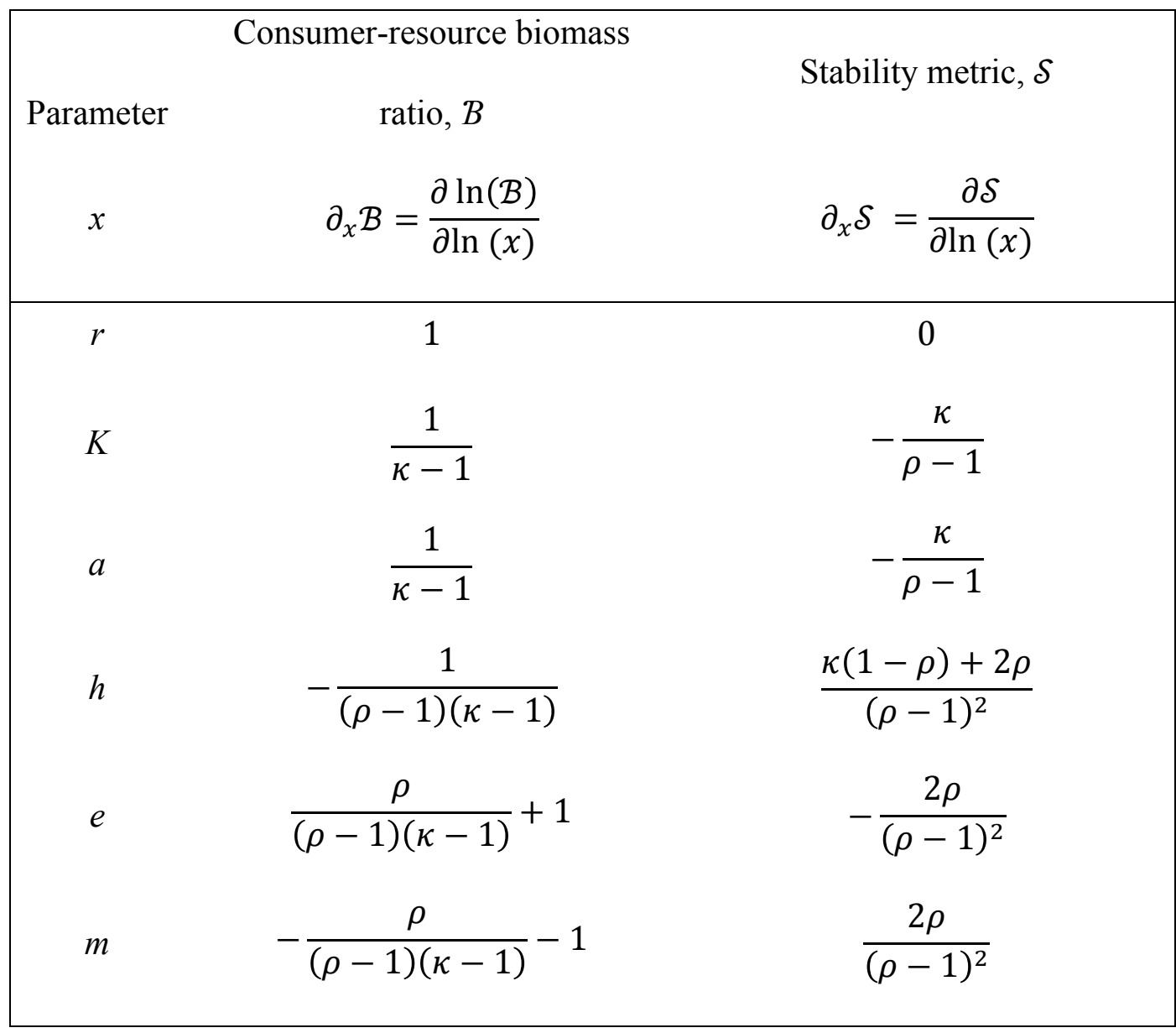




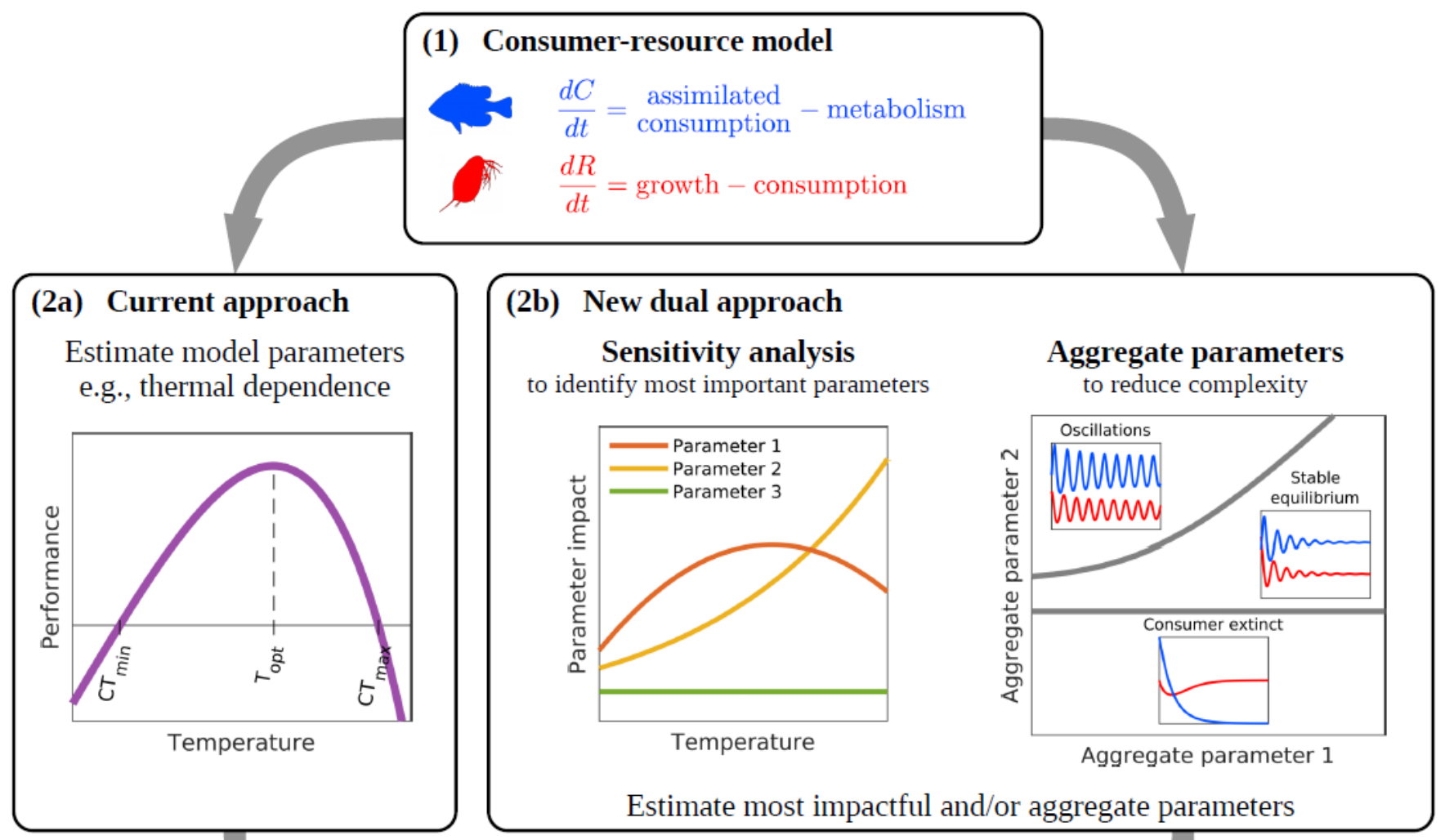

961

(3) Predict community properties

Biomass distribution

Temporal stability
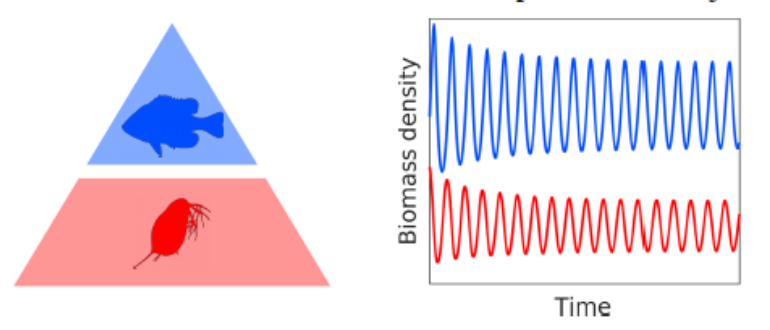

962

963 Figure 1. Illustration of the current and new dual approaches to predict the impact of global

964 change drivers on community properties. 1) Predictions require a consumer-resource model;

965 the Rosenzweig-MacArthur model (Rosenzweig \& MacArthur 1963) or its bioenergetic

966 equivalent (Yodzis \& Innes 1992) have been used most commonly for ectotherm consumer-

967 resource pairs. 2a) The current approach is to experimentally measure the response of

968 parameters along an environmental gradient, e.g. the thermal dependence of the resource

969 population maximal growth rate with critical temperatures, $C T_{\min }, C T_{\max }$, and the thermal 
970 optimum, $T_{\text {opt }}$. These measurements are used to parameterise the model. Importantly, not all

971 parameters are measured, but rather those which are considered significant (e.g. consumer

972 feeding and metabolic rates for warming-stability relationships). Assuming the remaining

973 parameter values, the model is then used to generate predictions. 2b) Our new dual approach

974 aims to increase the accuracy of predictions and facilitate their comparison. First a sensitivity

975 analysis determines which parameters have the greatest relative impact on the community

976 property of interest along the environmental gradient. Then, aggregate parameters which

977 represent biologically measurable quantities are used to express all sensitivities and

978 determine the dynamics. Collapsing analyses to the two aggregate parameters reduces

979 complexity and increases mechanistic tractability. This facilitates the choice of which

980 parameters need to be measured. 3) Through the empirical determination of the most

981 appropriate parameters (either from the original model parameters of the aggregate

982 parameters themselves) and the reduction in the number of measurements required, prediction

983 accuracy improves. The advantages of the new dual approach are twofold. First, as the

984 sensitivity analysis will have identified the most impactful parameters, the source of

985 divergence in predictions can be isolated. Second, the aggregates represent standardised

986 measurable population-level indicators across systems, making theoretical or empirical

987 predictions directly comparable. 
(a) Unimodal parameterisation

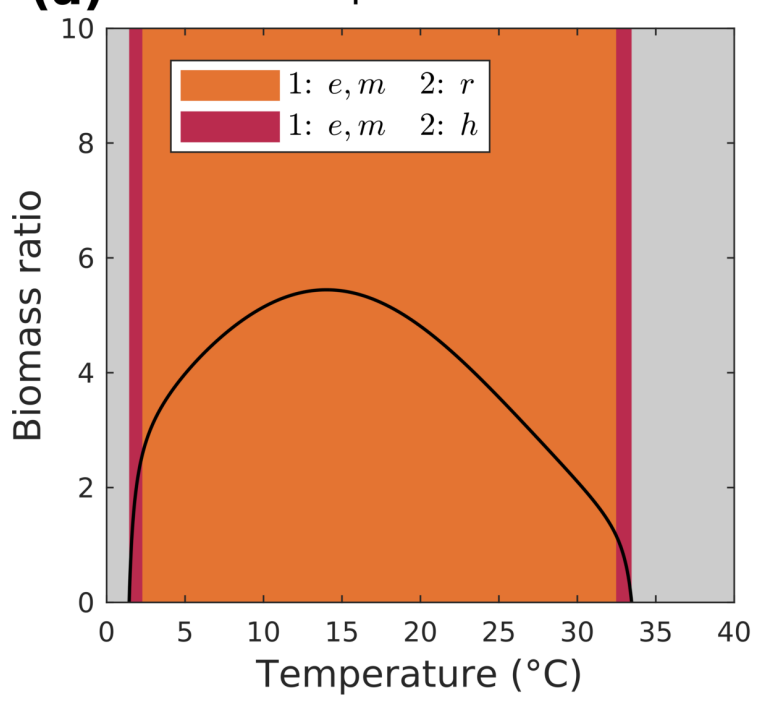

(c)

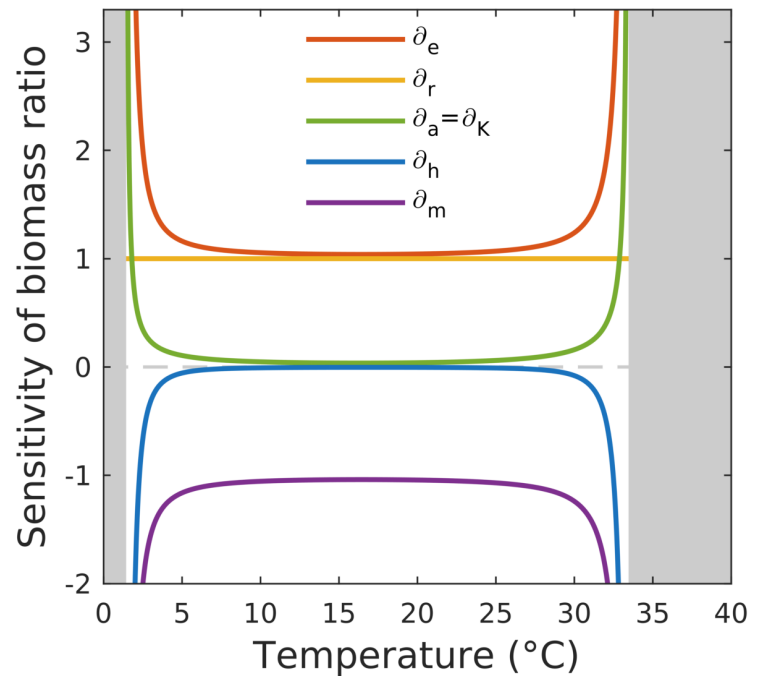

(b) Monotonic parameterisation

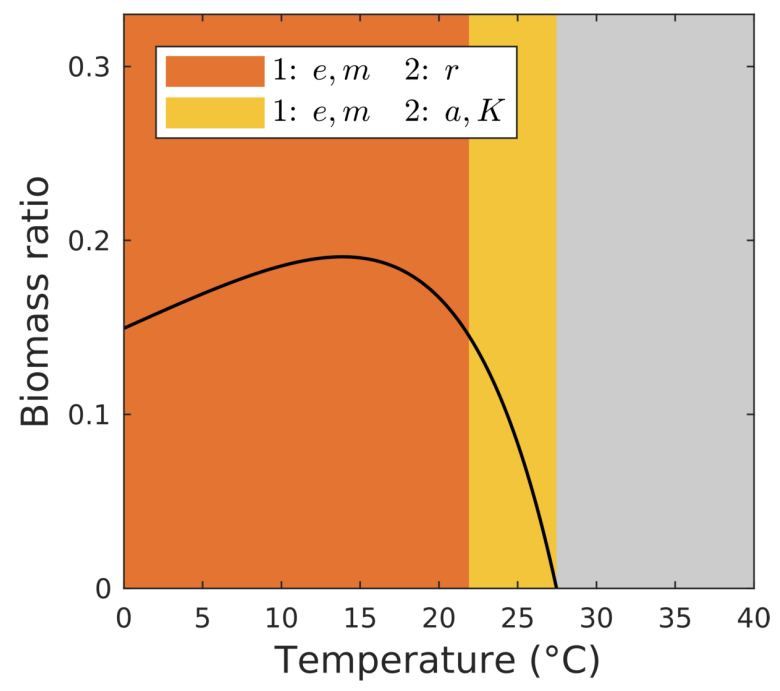

(d)

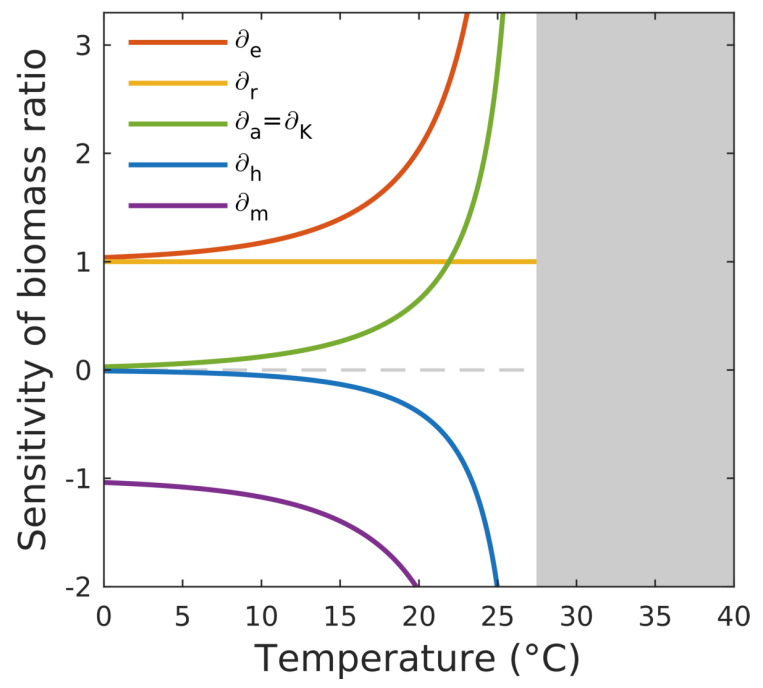

989

990 Figure 2. Consumer-resource biomass ratios for the (a) unimodal and (b) monotonic

991 parametrisations along the temperature gradient. Feasible temperature ranges are constrained

992 by the condition of positive biomass densities for both consumer and resource (grey areas

993 correspond to consumer extinction). The different background colours correspond to different

994 elasticity rankings of model parameters (see legend). Panels (c) and (d) provide the values of

995 the six parameter elasticities along the temperature gradient for the unimodal and monotonic

996 parameterisations, respectively. 
(a)

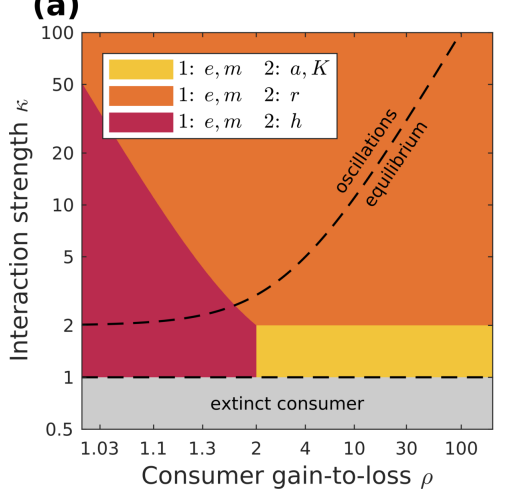

(b) Unimodal parameterisation

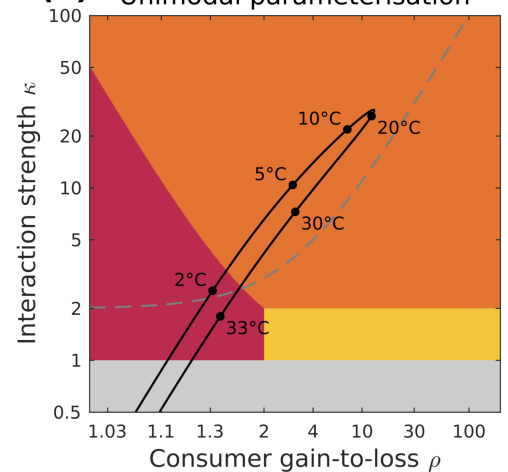

(c) Monotonic parameterisation

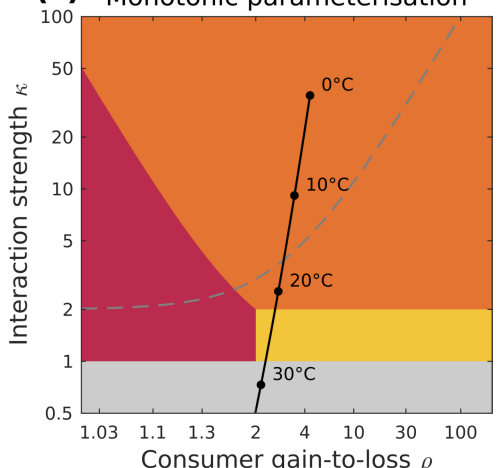

999 Figure 3. (a) Biomass ratio elasticity rankings in the $\rho-\kappa$ plane. The plane is split into regions

1000 (different colours) which correspond to different parameters having the top-two largest

1001 elasticities. These regions have been derived from the analytic expressions of the elasticities

1002 (Table 1). $e$ and $m$ elasticities always rank first. Close to consumer extinction $h$ ranks second

1003 highest at low $\rho(\rho<2$, red region) and $a$ and $K$ at higher $\rho(\rho>2$, yellow region). $r$ ranks

1004 second highest far from consumer extinction (orange region). The plane includes the

1005 feasibility boundary $(\kappa=1)$ and the Hopf bifurcation (dotted curve splitting the plane into

1006 stable equilibrium and oscillations). For the (b) unimodal and (c) monotonic

1007 parameterisations from the literature, the thermal dependencies of $\rho=\frac{e}{m h}$ and $\kappa=\frac{K}{R_{S}}$ were

1008 calculated. This yielded a trajectory for each parameterisation (solid black line). The paths of

1009 the trajectories demonstrate the elasticity of the biomass ratio along the temperature gradient

1010 for each parameterisation. 
(a) Vucic-Pestic et al. 2011
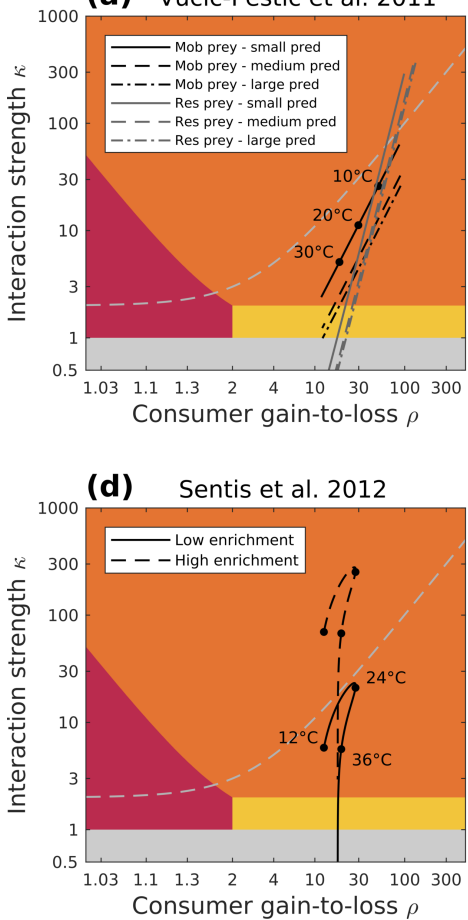

(b) Fussmann et al. 2014

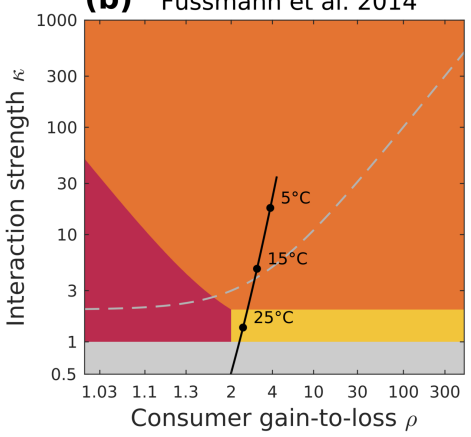

(e) Uszko et al. 2017

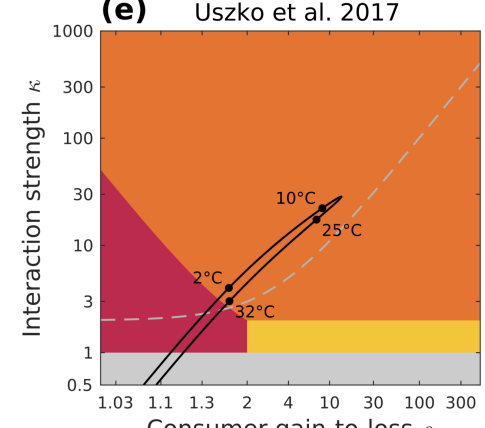

(c) Binzer et al. 2016

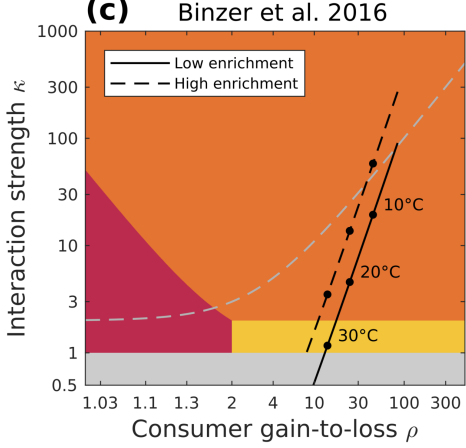

(f) Archer et al. 2019

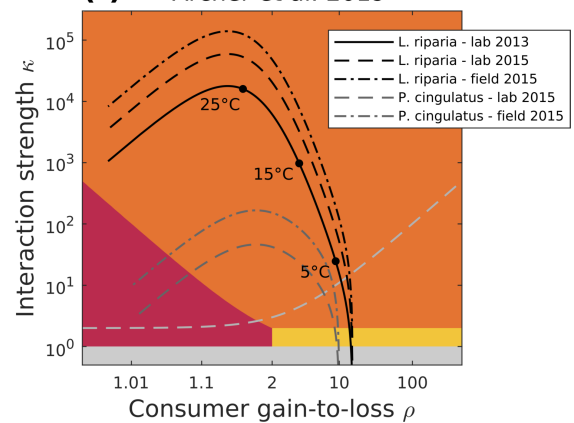

1012 Figure 4. Trajectories of the six empirical temperature parameterisations in the $\rho-\kappa$ plane: a)

1013 Vucic-Pestic et al. (2011) with six different experiments - three predator size-classes and two

1014 types of prey, b) Fussmann et al. (2014), c) Binzer et al. (2016) with two levels of

1015 enrichment, d) Sentis et al. (2012), with two levels of enrichment, e) Uszko et al. (2017), f)

1016 Archer et al. (2019) with two prey types and three measurements. (a), (b) and (c) have

1017 monotonic thermal dependences for $a, m$ (increasing) and $h, K$ (decreasing), and a constant $e$.

1018 (d) has a unimodal thermal performance curve for $a$ (hump-shaped), constant $e$ and $K$,

1019 monotonic $h$ (decreasing) and $m$ (increasing). (e) has a unimodal (U-shaped) $h$ and $a$ (hump-

1020 shaped) thermal dependence, constant $e$ and monotonic $K, m$ (increasing). (f) has monotonic $a, K, m, e$ (increasing) and $h$ constant. All parameter values are detailed in SI 4 . The coloured regions demonstrate the different biomass ratio sensitivity rankings (see legend in Fig. 3a).

1023 The trajectories (solid black lines) for each parameterisation are derived from calculating the 1024 thermal dependence of $\rho=\frac{e}{m h}$ and $\kappa=\frac{K}{R_{S}}$ (see Temperature dependencies and parameterisations for details). 
(a) Unimodal parameterisation

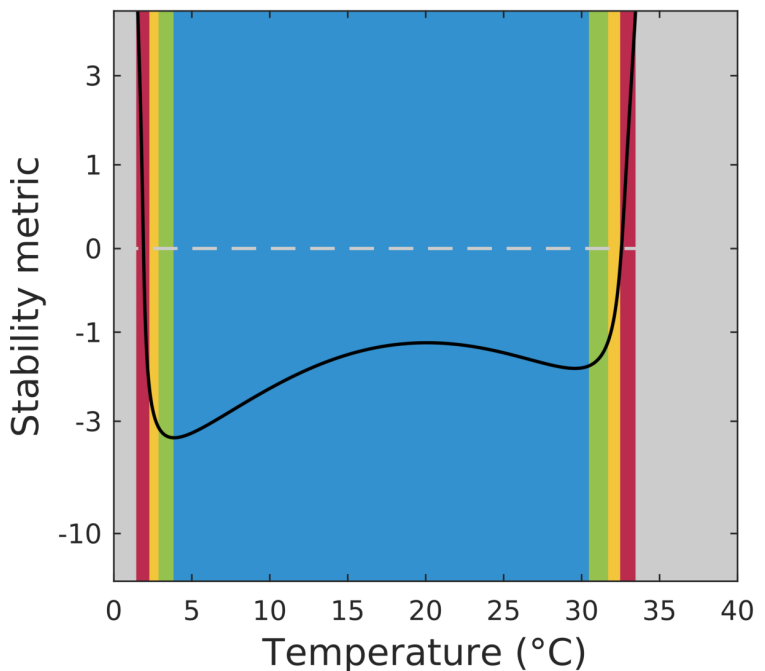

(c)



(b) Monotonic parameterisation

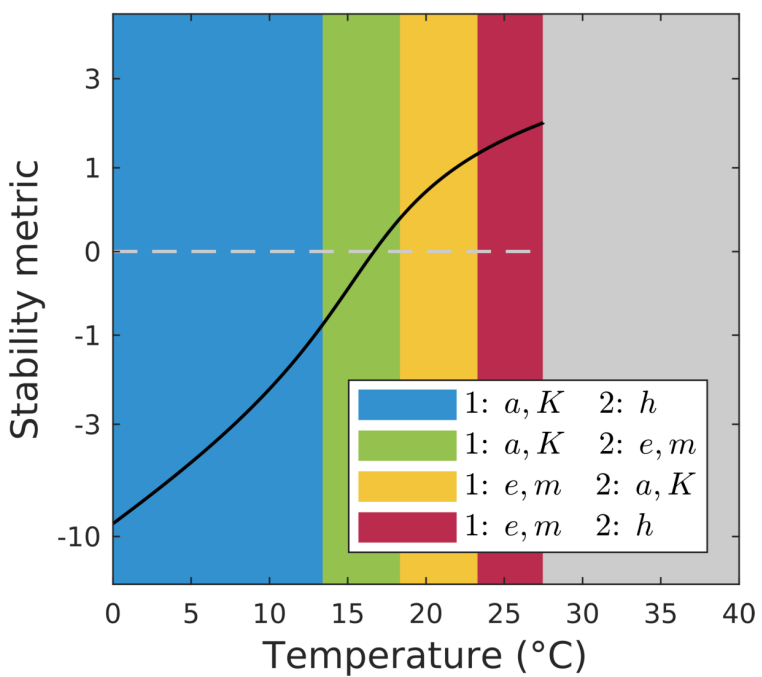

(d)

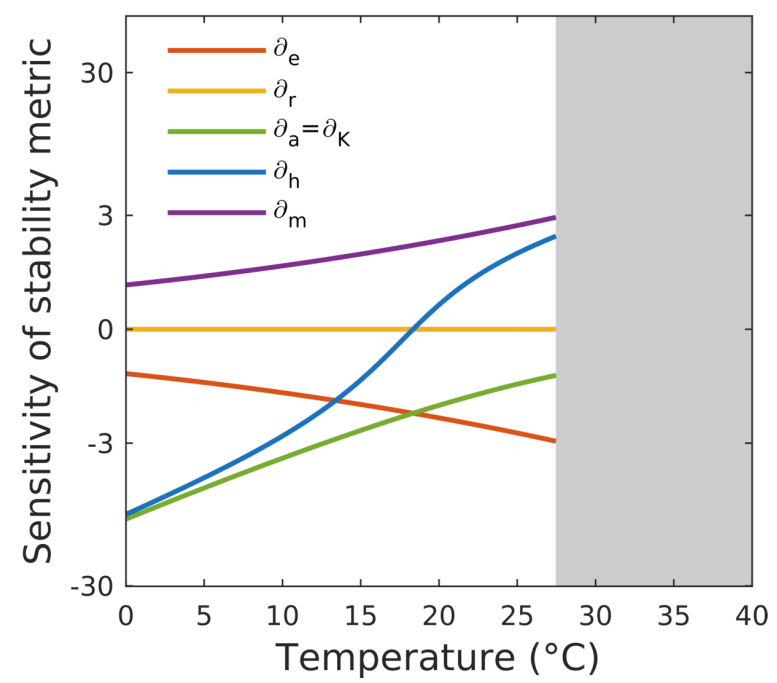

1028 Figure 5. The thermal dependence of the stability metric, $\mathcal{S}$, for the (a) unimodal and (b)

1029 monotonic parameterisations. $\mathcal{S}>0$ corresponds to stable dynamics, $\mathcal{S}<0$ to oscillations. $\mathcal{S}$ $1030=0$ (dotted line) corresponds to the Hopf bifurcation. The coloured temperature ranges

1031 highlight regions of different sensitivity rankings. For temperatures beyond the community

1032 feasibility boundaries the areas are greyed out. In (c) and (d) the sensitivity to each parameter

1033 is plotted along the temperature gradient for the unimodal and monotonic parameterisations, 1034 respectively. 
(a)

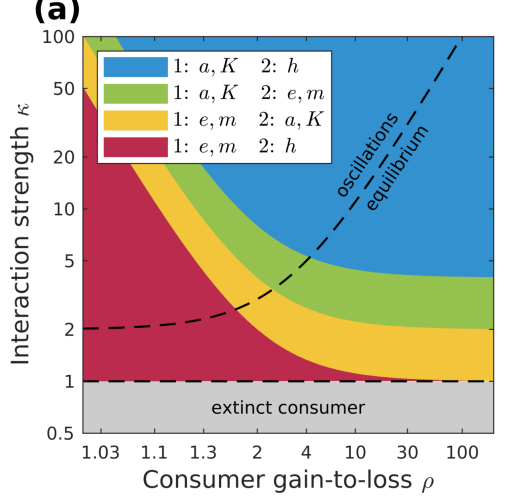

(b) Unimodal parameterisation

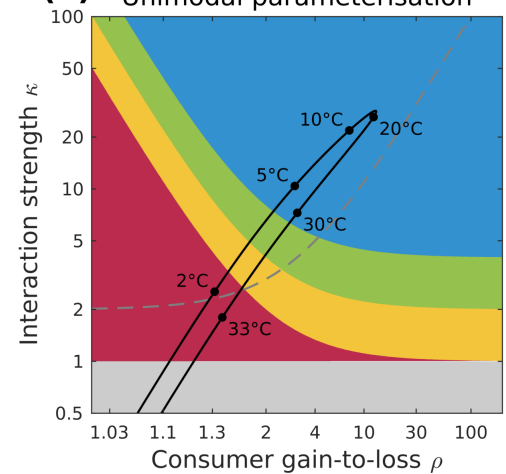

(c) Monotonic parameterisation

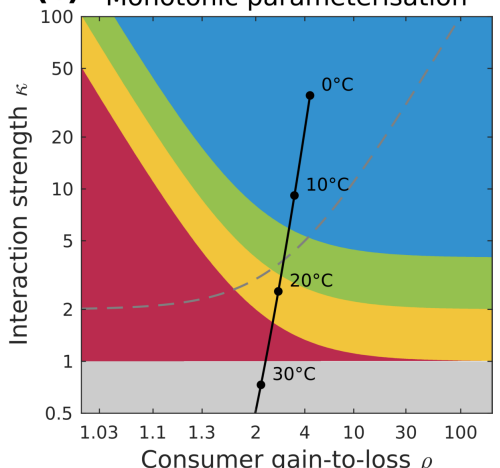

1036 Figure 6. (a) Stability metric sensitivity rankings in the $\rho-\kappa$ plane. The plane is split into regions (different colours) which correspond to different parameters having the top-two

1038 largest sensitivities. These regions have been derived from the analytic expressions of the

1039 elasticities (Table 1). $e$ and $m$ rank first close to consumer extinction; $h$ ranks second highest

1040 closest to consumer extinction (red region). The sensitivity of stability to $a$ and $K$ increases

1041 moving away from consumer extinction. $a$ and $K$ sensitivity ranks second (yellow region),

1042 and then first moving further away. Initially $e$ and $m$ rank second (green region) before $h$

1043 becomes significant (blue region). The plane includes the feasibility boundary $(\kappa=1)$ and the

1044 Hopf bifurcation (dotted curve splitting the plane into stable equilibrium and oscillations).

1045 For the (b) unimodal and (c) monotonic parameterisations from the literature, the thermal

1046 dependencies of $\rho=\frac{e}{m h}$ and $\kappa=\frac{K}{R_{S}}$ were calculated. This yielded a trajectory for each

1047 parameterisation (solid black line). The paths of the trajectories demonstrate the dynamical

1048 regime and the sensitivity of the stability metric along the temperature gradient for each 1049 parameterisation. 
(a) Vucic-Pestic et al. 2011
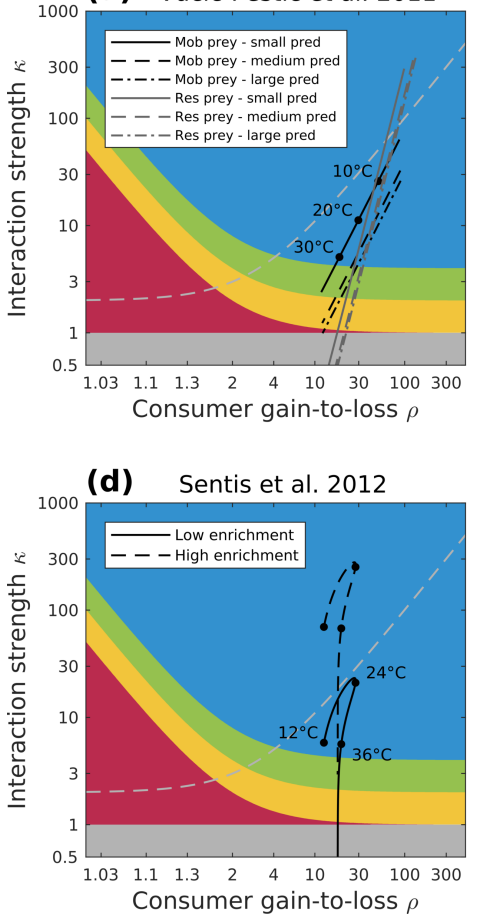

(b) Fussmann et al. 2014

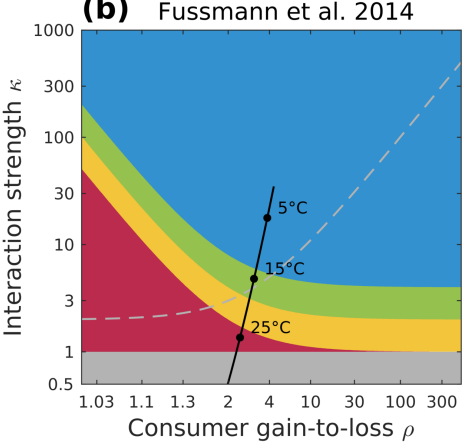

(e) Uszko et al. 2017

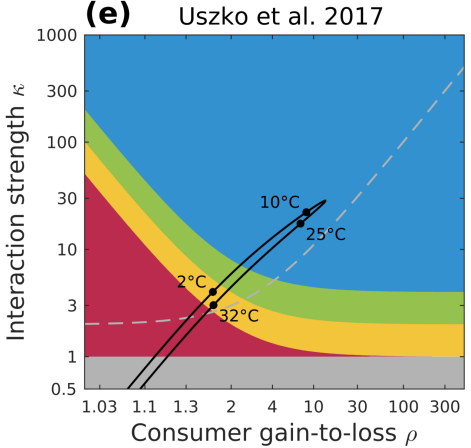

(c) Binzer et al. 2016
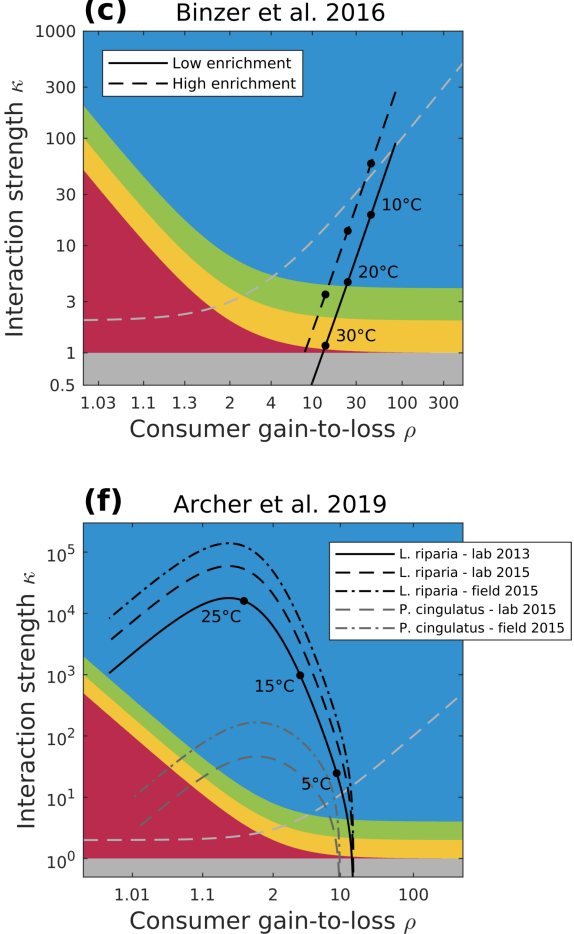

1052 Figure 7. Trajectories of the six empirical temperature parameterisations in the $\rho$ - $\kappa$ plane: a)

1053 Vucic-Pestic et al. (2011) with six different interaction experiments - three predator size1054 classes and two types of prey, b) Fussmann et al. (2014), c) Binzer et al. (2016) with two 1055 levels of enrichment, d) Sentis et al. (2012), with two levels of enrichment, e) Uszko et al. (2017), f) Archer et al. (2019) with two prey types and three measurements. (a), (b) and (c) have monotonic thermal dependences for $a, m$ (increasing) and $h, K$ (decreasing), and a constant $e$. (d) has a unimodal thermal performance curve for $a$ (hump-shaped), constant $e$ and $K$, monotonic $h$ (decreasing) and $m$ (increasing). (e) has a unimodal (U-shaped) $h$ and $a$ 1060 (hump-shaped) thermal dependence, constant $e$ and monotonic $K, m$ (increasing). (f) has monotonic $a, K, m, e$ (increasing) and $h$ constant. All parameter values are detailed in SI 4.

1062 The coloured regions demonstrate the different biomass ratio sensitivity rankings (see legend 1063 in Fig. 6a). The trajectories (solid black line) for each parameterisation are derived from 1064 calculating the thermal dependence of $\rho=\frac{e}{m h}$ and $\kappa=\frac{K}{R_{S}}$. Therefore, trajectories do not change with the variable of interest; the sensitivity regions do. 